\title{
Quantum noise in the mirror-field system: A field theoretic approach
}

\author{
Jen-Tsung Hsiang, Tai-Hung Wu, and Da-Shin Lę* \\ Department of Physics, National Dong-Hwa University, Hua-lien, Taiwan, R. O. C. \\ Sun-Kun King \\ Academia Sinica, Institutes for Astronomy and Astrophysics, Taipei, Taiwan, R.O.C. \\ Chun-Hsien $\mathrm{Wu}$ \\ Department of Physics, Soochow University, Taipei, Taiwan, R.O.C.
}

(Dated: June 16, 2022)

\begin{abstract}
We employ the field theoretic approach to study the quantum noise problem in the mirror-field system, where a perfectly reflecting mirror is illuminated by a single-mode coherent state of the massless scalar field. The associated radiation pressure is described by a surface integral of the stress-tensor of the field. The read-out field is measured by a monople detector, form which the effective distance between the detector and mirror can be obtained. In the slow-motion limit of the mirror, we are able to identify various sources of quantum noise that lead to uncertainty of the read-out measurement. Since the mirror is driven by radiation pressure, the sources of noise, other than the shot nose given by the intrinsic fluctuations of the incident state, may also result from random motion of mirror due to radiation pressure fluctuations and from modified field fluctuations induced by the displacement of the mirror. Correlation between different sources of noise can be established as the consequence of interference between the incident field and the reflected field out of the mirror in the read-out measurement. The overall uncertainty is found to decrease (increase) due to the negative (positive) correlation. In the case of negative correlation, the uncertainty can be lowered than the value predicted by the standard quantum limit. We also examine the validity of the particle number approach, which is often used in quantum optics, and compared its results with those given by the field theoretical approach. Finally we discuss the backreaction effects, induced by the radiation pressure, that alter the dynamics of the mean displacement of the mirror, and we argue this backreaction can be ignored for a slowly moving mirror.
\end{abstract}

PACS numbers: 03.70.+k, 05.40.-a, 12.20.Ds, 42.50.Dv, 42.50.Lc

*Electronic address: dslee@mail.ndhu.edu.tw 


\section{INTRODUCTION}

The measurement of a very weak force such as the detection of gravitational wave demands a very high resolution interferometer. The role of the interferometer is to transform small variation of the relative spatial separation of the mirrors between two arms into variation of the output photons. Quantum noise presumably places a limitation on accuracy of the measurement [1].

In quantum optics, the discussion related to quantum noise of radiation fields on the mirror has been mostly based on the photon-number approach. It is argued by Caves [3, 4] that quantum noise in a laser interferometer may come from the quantum nature of the light directly via photon number fluctuations (shot noise) or indirectly via random motion of the mirror due to a fluctuating force (radiation pressure fluctuations). Minimizing the total uncertainty from two sources of noise, with an assumption of no correlation between them, may give the standard quantum limit (SQL), when an input power of the light is appropriately tuned. Additionally, Caves further suggests that injecting squeezed vacuum state into the unused port of an interferometer should lead to the SQL with a lower optimum input power [3], as compared with the standard interferometer [4]. This seminal work also shows the manipulated quantum fluctuations of the electromagnetic fields are responsible for both the radiation-pressure forces and the fluctuations of the photon number. Then, an inquiry into the assumption of no correlation between these two noise sources arises. In particular, the presence of negative correlations may lead to the uncertainly even below the SQL. To establish their correlations requires an unified scheme that has been proposed by Loudon [7]. The judicious use of the correlated squeezed state is proposed to establish the above correlations with which to possibly push the sensitivity of the interferometer beyond the SQL [10]. The redesign of an interferometer by adding some optical components to either manipulate the read-outs or modify the dynamics of the test mass, such as in the "optical bar" [11] or the "optical spring" [12] scheme, is also possibly to give non-zero correlations in order to beat the SQL.

The coupling between the mirror and the radiation field can be obtained by considering the mirror as a reflector that imposes boundary conditions of the field on the surface of the mirror. In $3+1$ dimensions, field quantization is treated perturbatively for a slowly moving, perfectly reflecting mirror by Vilenkin and Ford [13], with the boundary conditions of the field on the surface of the mirror at all times. They then explore the backreaction dissipative

effect on a moving mirror, induced by vacuum fluctuations of the field. Later a corresponding Langevin equation was derived by two of us [14] with the method of influence functional, in which the accompanying noise, manifested from vacuum fluctuations is taken into account. Thus, the effects of the backreaction dissipation and its accompanying noise can be related by 
the underlying fluctuation-dissipation relation. Unruh [8] extended the quantization scheme to the interferometer case where the field propagates toward a mirror and then acquires a time-dependent phase shift after reflecting off the mirror. Because of the moving boundary, the phase shift in general depends on the mirror's motion, but can be obtained by assuming slow-motion of the mirror. Similar phase shift was also introduced by Kimble et al. [9], and it has been proposed to improve performance of the laster interferometer, particularly, in [12].

Here we adopt the field quantization scheme similar to Unruh's proposal to investigate the problem of quantum noise and its associated correlation in a simpler configuration, shown in Fig. 1. The basic idea is to consider a free, perfectly reflecting mirror. A coherent state of the quantum scalar field is normally shined on the mirror's surface, and exerts a pressure force on the mirror. It drives the mirror into motion, which in turn leads to variation of the reflected field. Then the read-out field is measured by a standard monopole detector, which is placed somewhere between the mirror and the field source. Since the radiation pressure is described by a surface integral of the stress-tensor of the scalar field, if the input quantum state is not an eigenstate of the operator associated with the response function of the detector or the stress-tensor, the resulting measurement will exhibit fluctuations.

The goal of this paper is two-fold. First of all, we lay out a field-theoretic approach by which the effective distance between the detector and the mirror can be decoded from the read-out fields. In this simpler system, the sources of the uncertainty in the read-outs can be easily identified in the slow motion limit when the mirror is driven by radiation pressure of the incident field. Thus reduction in uncertainty of this effective distance is of essence to improve the sensitivity of the interferometer. When the incident field is in the single-mode coherent state, it will be shown that the particle number approach, typically used in quantum optics, is equivalent to this field theoretic approach in the late-time limit with a large number of the scalar particles (photon). We will elaborate the previously mentioned approximations later. In addition to shot noise and radiation pressure fluctuations, a new source of noise comes from modified field fluctuations. It is induced by the mean displacement of the mirror as a result of the radiation pressure. Its effect can be systematically examined in the field theoretic approach. Secondly, the emphasis is put on how correlation among all sources of quantum noise associated with the radiation field, can emerge when the interference of the incoming field and the reflected filed by the mirror are taken into account. We further examine the effects of correlation on possible reduction of uncertainty in the read-outs of the monopole detector. We show an example in which the overall uncertainty can be suppressed to beat the standard quantum limit.

Our presentation is organized as follows. In Sec. II], we introduce the quantization scheme of a massless scalar field subject to boundary conditions on a free, perfectly reflecting mirror 
when it undergoes slow motion. The motion of the mirror arises from radiation pressure of the scalar field. A monopole detector is introduced to measure the readout field. We then identify the sources of quantum noise from the fluctuations of the readouts in Sec. III with an emphasis on establishing correction between them. The effects of correlation are further studied in Sec. IV] for possibility of reducing overall quantum noise. In Sec. V the Langevin equation of the mirror, including backreaction from the radiation field, is derived, and we show that this effect is negligible for slow motion. Conclusions are drawn in Sec VI.

The Lorentz-Heaviside units and the convention $\hbar=c=1$ will be used unless otherwise mentioned. The signature of the metric is $\operatorname{diag}\left\{\eta_{\mu \nu}\right\}=(+1,-1,-1,-1)$.

\section{FIELD QUANTIZATION AND DETECTOR THEORY}

We consider a free mirror of perfect reflectance is illuminated by a single-mode coherent laser, which propagates in a direction normal to the mirror. The radiation pressure of the laser will drive the mirror into motion, and the field, bounced off the mirror, can be measured by a detector. For simplicity and without loss of generalization, the radiation field is represented by a massless scalar field $\phi$ to suppress complexity caused by the vector nature of the electromagnetic field. The mirror, with mass $m$, is placed at $z=L$. Its lateral dimensions in the $x, y$ directions are large compared to wavelength of the incoming state, and the cross sectional area is $\mathcal{A}_{\|}$. We use $\mathbf{x}_{\|}$to denote directions normal to the $z$ axis. The incident field is a plane wave propagating along the positive $z$ direction toward the mirror. The boundary condition of the field on the mirror surface is then given by

$$
\phi\left(\mathbf{x}_{\|}, L+q(t), t\right)=0
$$

where $q(t)$ is the displacement of the mirror from its original position at $z=L$ due to radiation pressure. The approximate solution to the field equation subject to this moving boundary condition in the region of $z<L$ is [8]

$$
\phi=\phi^{+}+\phi^{-}
$$

where the positive-frequency solution $\phi^{+}$is given by

$$
\phi^{+}(\mathbf{x}, t)=\int^{\prime} \frac{d^{3} k}{(2 \pi)^{3 / 2}} \frac{1}{\sqrt{2 \omega}} a_{k} \mathcal{U}_{\mathbf{k}}(\mathbf{x}, t)
$$

with

$$
\mathcal{U}_{\mathbf{k}}(\mathbf{x}, t)=e^{i \mathbf{k}_{\|} \cdot \mathbf{x}_{\|}-i \omega t}\left[e^{i k_{z} z}-e^{-i k_{z}\left(z-2 L-2 q\left(t_{R}\right)\right)}\right] .
$$

The negative-frequency solution $\phi^{-}$is the Hermitian conjugate of the positive-frequency solution $\phi^{+}$, that is, $\phi^{-}=\left(\phi^{+}\right)^{\dagger}$. The prime over the integrals of $\mathbf{k}$ means that the modes 
with $k_{z}<0$ are excluded because these modes propagate in the negative $z$ direction and will not interact with the mirror in the $z<L$ region. Here we have assumed that the mirror undergoes slow motion $(\dot{q} \ll 1)$. Since the incoming wave is a right-moving plane wave along the $z$ direction, the left-moving reflected wave will receive a time-dependent phase shift due to the motion of the mirror. The phase shift of the reflected field at time $t$ and position $z$ depends on the mirror's position at an earlier time $t_{R}=t-\left|L+q\left(t_{R}\right)-z\right|$ if we take finite lapse of propagation into consideration. When the displacement is small, we may take the retarded time $t_{R}$ as $t_{R}=t-|L-z|$ in the leading order approximation.

Due to small displacement approximation, the force acting on the mirror by the fields can be approximated by the area integral of the $z z$ component of the energy-momentum stress tensor of the scalar field, evaluated at the mirror's original position $z=L$,

$$
F_{0}(t)=\int d \mathcal{A}_{\|} T_{z z}\left(\mathbf{x}_{\|}, L, t\right),
$$

where the $z z$ component of the energy-momentum stress tensor takes the form

$$
T_{z z}\left(\mathbf{x}_{\|}, z, t\right)=\frac{1}{2}\left[\left(\partial_{t} \phi\right)^{2}+\left(\partial_{z} \phi\right)^{2}-\left(\partial_{\mathbf{x}_{\|}} \phi\right)^{2}\right] .
$$

Hereafter we will use $d \mathcal{A}_{\|}$to denote the area element normal to the $z$ direction. Thus, if the mirror is at rest at $z=L$ for $t<0$, then the displacement operator due to the action of radiation pressure of the incident state is formally given by

$$
q(t)=\frac{1}{m} \int_{0}^{t} d s \int_{0}^{s} d s^{\prime} \int d \mathcal{A}_{\|} T_{z z}\left(\mathbf{x}_{\|}, L, s^{\prime}\right) .
$$

Since the energy-momentum stress tensor is quadratic in the field operator $\phi$, the displacement operator is not well-defined. A proper renormalization of mirror's position operator can be done by absorbing the vacuum expectation value of the displacement operator into the initial position $L$, namely,

$$
q(t)+L=q(t)-\langle q(t)\rangle_{0}+\left(L+\langle q(t)\rangle_{0}\right)=q_{r}(t)+L_{r} .
$$

This prescription is physically sensible since when the mirror is placed at its initial position, the vacuum fluctuations of the field have already interacted with the mirror before the incoming state impinges on it. Thus, the renormalized displacement operator $q_{r}(t)$ now takes the form

$$
q_{r}(t)=\frac{1}{m} \int_{0}^{t} d s \int_{0}^{s} d s^{\prime} \int d \mathcal{A}_{\|}\left\{T_{z z}\left(\mathbf{x}_{\|}, L, s^{\prime}\right)-\left\langle T_{z z}\left(\mathbf{x}_{\|}, L, s^{\prime}\right)\right\rangle_{0}\right\} .
$$

Here the backreactions of the field is ignored. This backreaction can be incorporated in the Langevin equation, Eq. (43), and is found negligible for a slowly moving mirror. To 


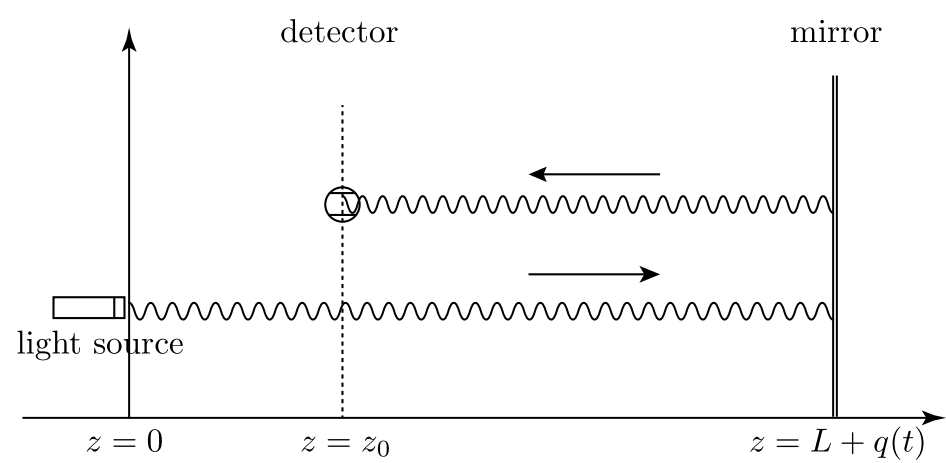

FIG. 1: Schematic diagram of the field-mirror system.

avoid cluttering the notion, we will suppress the subscript $r$ of the renormalized quantities hereafter.

To measure the outcomes of the scalar field, we employ a monopole detector. We assume that the detection processes is based on the stimulated transition of the detector due to coupling between the scalar field and the monopole moment $m(t)$ of the detector,

$$
\int^{t} d s m(s) \phi(\mathbf{x}, s)
$$

In the standard detector theory, the dynamics of the detector is governed by some timeindependent Hamiltonian $H_{D}$. Assume that the detector is a two-level system with eigenenergies $E_{1}, E_{2}$ such that $H_{D}\left|E_{1,2}\right\rangle=E_{1,2}\left|E_{1,2}\right\rangle$ and $E_{1}<E_{2}$. In the interaction picture the monopole moment evolves in time as

$$
m(t)=e^{i H_{D} t} m(0) e^{-i H_{D} t} .
$$

Then by first-order perturbation theory, the transition rate between these two states of the detector is given by

$$
P\left(E_{1} \rightarrow E_{2}\right)=\left|\left\langle E_{1}|m(t)| E_{2}\right\rangle\right|^{2} \times \Pi_{\phi}\left(E_{2}-E_{1}\right) .
$$

The response function $\Pi_{\phi}$ is defined by

$$
\begin{aligned}
\Pi_{\phi}(E) & =\int d s \int d s^{\prime} e^{-i E\left(s-s^{\prime}\right)}\left\langle\phi^{-}(\mathbf{x}, s) \phi^{+}\left(\mathbf{x}, s^{\prime}\right)\right\rangle_{\bar{\alpha}} \\
& =\delta(E-\bar{\omega}) \int d s\left\langle\phi^{-}(\mathbf{x}, s) \phi^{+}(\mathbf{x}, s)\right\rangle_{\bar{\alpha}},
\end{aligned}
$$

where we have assumed that the incident field is in a single-mode coherent state $|\bar{\alpha}\rangle$ with frequency $\bar{\omega}$. Eq. (9) implies that we may construct an integral quantity by

$$
I\left(z_{0}, t ; L+q\right)=\int_{0}^{t} d s \int d \mathcal{A}_{\|} S\left(\mathbf{x}_{\|}, z_{0}, s ; L+q\right),
$$


with

$$
S(x ; L+q)=\phi^{-}(x) \phi^{+}(x), \quad \text { and } x=\left(t, \mathbf{x}_{\|}, z_{0}\right),
$$

where the detector is placed at $z=z_{0}$ and the position of the mirror is located at $z=L+q(t)$. The quantity $I$ is a measure that generically summarizes accumulated measurement results over finite time duration. Integration over an area of the mirror size illustrates the situation that if the size of the detector is much greater than $2 \pi / \bar{k}_{\|}$of the incoming coherent mode, then the measurements have been averaged in the lateral spatial direction. Then in terms of the mode functions of the scalar field, $I\left(z_{0}, t ; L+q\right)$ can be expressed as

$$
I\left(z_{0}, t ; L+q\right)=\int_{0}^{t} d s \int d \mathcal{A}_{\|} \int^{\prime} \frac{d^{3} k}{(2 \pi)^{\frac{3}{2}}} \int^{\prime} \frac{d^{3} k^{\prime}}{(2 \pi)^{\frac{3}{2}}} \frac{1}{\sqrt{2 \omega}} \frac{1}{\sqrt{2 \omega^{\prime}}} \mathcal{U}_{\mathbf{k}}^{*}\left(\mathbf{x}_{\|}, z_{0}, s\right) \mathcal{U}_{\mathbf{k}^{\prime}}\left(\mathbf{x}_{\|}, z_{0}, s\right) a_{\mathbf{k}}^{\dagger} a_{\mathbf{k}^{\prime}} .
$$

For the coupled mirror-field system, we notice that they are at least two sources that will contribute to the uncertainty associated with $I$. One directly results from quantum fluctuations of the incoming field, but the other indirectly comes from the stochastic motion of the mirror, which in turn is the consequence of radiation-pressure fluctuations of the field. In other words, the quantum fluctuations of the incoming field will drive the mirror into stochastic motion, and next the moving mirror will modify the reflected field in a complicated way, depending on mirror's motion. If we use the a detector to measure field variation at $z=z_{0}<L$, the detector will see combined effects from the incoming and the reflected field. It will be shown later that the interference between the right-moving (incoming) and left-moving (outgoing) field leads to the result that the phase of the expectation value of the operator $I\left(z_{0}, t ; L\right)$ depends on the effective distance between the mirror and the detector. In addition, this interference effect is essential to establish correlation between different sources of quantum noise. This phenomenon gives a hope to reduce overall quantum noise.

\section{QUANTUM NOISE}

Since the mirror is driven by the radiation pressure of a single-mode coherent state with the frequency $\bar{\omega}$, the assumption of slow motion of the mirror can be realized by

$$
\bar{\omega} q(t) \ll 1, \quad q(t) / L \ll 1 .
$$

This suggests that we may formally expand the field operators, $\phi^{ \pm}(x ; q+L)$ and $I\left(z_{0}, t ; q+L\right)$,

in a power series of the the displacement operator $q(t)$. The operator ordering can be quite ambiguous in this case. However, it can be shown that the leading-order result is independent of operator ordering. Thus, for example, up to the second order in $q$, we may just expand $I$ into

$$
I\left(z_{0}, t ; q+L\right)=I\left(z_{0}, t ; L\right)+q\left(t_{\mathrm{ret}}\right) \partial_{L} I\left(z_{0}, t ; L\right)+\frac{1}{2} q^{2}\left(t_{\mathrm{ret}}\right) \partial_{L}^{2} I\left(z_{0}, t ; L\right)+\mathcal{O}\left(q^{3}\right),
$$


irrespective of the operator ordering. Apparently, the first term represents the measurement performed with respect to the mirror's original position. The other terms can be understood as corrections which result from the mirror's motion due to radiation pressure.

The single-mode coherent state of the background field can be obtained by applying the displacement operator $D(\bar{\alpha})$ on the corresponding vacuum state,

$$
|\bar{\alpha}\rangle=D(\bar{\alpha})|0\rangle,
$$

where the displacement operator $D(\bar{\alpha})$ is defined by

$$
D(\bar{\alpha})=\exp \left[\bar{\alpha} a_{\bar{\omega}}^{\dagger}-\bar{\alpha}^{*} a_{\bar{\omega}}\right] .
$$

Then, for a fixed position of the mirror, the signal is given by the expectation value of $\left\langle I\left(z_{0}, t ; L\right)\right\rangle_{\bar{\alpha}}$, which is obtained from Eq. (11) by setting $q=0$

$$
\left\langle I\left(z_{0}, t ; L\right)\right\rangle_{\bar{\alpha}}=\frac{\mathcal{A}_{\|}}{(2 \pi)^{3}}|\bar{\alpha}|^{2} \frac{2}{\bar{\omega}} \sin ^{2} \bar{\omega}\left(L-z_{0}\right) \times t .
$$

The result depends on the relative distance between the detector plane and the mirror surface, which arises from the optical path difference between the right-moving (incoming) field and the left-moving (outgoing) field. If a free mirror, instead of a fixed one, is considered, the above result will be modified to account for the additional effect due to the mean displacement of the mirror. We further assume that the coherent state under consideration has a sufficiently large particle density $|\bar{\alpha}|^{2}$ so that in this state, the ratio of the operator's fluctuations over its expectation value, which is typically inversely proportional to $|\bar{\alpha}|^{2}$, is small. Thus, the variation of $I\left(z_{0}, t ; q+L\right)$ can be approximated by

$$
\begin{aligned}
\Delta I\left(z_{0}, t ; q+L\right)=\Delta I\left(z_{0}, t ; L\right)+ & \left\langle\partial_{L} I\left(z_{0}, t ; L\right)\right\rangle_{\bar{\alpha}} \Delta q\left(t_{\mathrm{ret}}\right)+\left\langle q\left(t_{\mathrm{ret}}\right)\right\rangle_{\bar{\alpha}} \Delta \partial_{L} I\left(z_{0}, t ; L\right) \\
+\left\langle q\left(t_{\mathrm{ret}}\right)\right\rangle_{\bar{\alpha}}\left\langle\partial_{L}^{2} I\left(z_{0}, t ; L\right)\right\rangle_{\bar{\alpha}} \Delta q\left(t_{\mathrm{ret}}\right)+\frac{1}{2}\left\langle q\left(t_{\mathrm{ret}}\right)\right\rangle_{\bar{\alpha}}^{2} \Delta \partial_{L}^{2} I\left(z_{0}, t ; L\right) & +\mathcal{O}\left(\Delta^{2}\right),
\end{aligned}
$$

if, given an operator $O$, its variation $\Delta O$ is defined as $\Delta O=O-\langle O\rangle_{\bar{\alpha}}$. This gives a leading-order effect of the noise. Later we will offer an order-of-magnitude estimate of the ignored terms. The first term of (16) arises from the intrinsic fluctuations of the incident state. For a large value of $|\bar{\alpha}|^{2}$, the particle number has relatively small uncertainty; thus according to 15 , the main contribution to the variation of $I\left(z_{0}, t ; L\right)$ for fixed $\bar{\omega}$ does not come from amplitude uncertainty, but is given by the phase fluctuations, as will be seen later in Eq. (30). It is thus associated with shot noise. The other terms may be understood as the induced effects due to either the position fluctuations of the mirror, or the modified field fluctuations as a result of the mean displacement of the mirror. Then the variation of 
$I\left(z_{0}, t ; q+L\right)$ allows us to define the overall uncertainty of the effective distance between the detector and the mirror,

$$
\Delta z=\frac{\Delta I\left(z_{0}, t ; q+L\right)}{\left|\left\langle\partial_{L} I\left(z_{0}, t ; L\right)\right\rangle_{\bar{\alpha}}\right|},
$$

as long as $|\bar{\omega} \Delta z|<1$. The normalization factor $\left\langle\partial_{L} I\left(z_{0}, t ; L\right)\right\rangle_{\bar{\alpha}}$ is to measure the change of $I\left(z_{0}, t\right)$ due to variation of the mirror's position. Control of the above uncertainty is essential to improve the sensitivity of the interferometer, in which the measurement of the separation between the mirrors and the beam splitter plays an important role.

To compute the square of the effective displacement uncertainty $(\Delta z)^{2}$ of the mirror, we may encounter terms such as $\left\langle S(x) S\left(x^{\prime}\right)\right\rangle-\langle S(x)\rangle\left\langle S\left(x^{\prime}\right)\right\rangle,\left\langle T_{z z}(x) T_{z z}\left(x^{\prime}\right)\right\rangle-$ $\left\langle T_{z z}(x)\right\rangle\left\langle T_{z z}\left(x^{\prime}\right)\right\rangle$, and their cross correlation terms. Since both $S(x)$ and $T_{z z}(x)$ are quadratic in field operators $\phi(x)$, they are not well-defined in the coincidence limit $x \rightarrow x^{\prime}$. We expect that the square of the effective displacement uncertainty should contain terms involving a product of four field operators. Thus it may be more transparent if we decompose such a product as follows,

$$
\begin{aligned}
\phi_{1} \phi_{2} \phi_{3} \phi_{4}=: \phi_{1} \phi_{2} \phi_{3} \phi_{4}:+: \phi_{1} \phi_{2}:\left\langle\phi_{3} \phi_{4}\right\rangle_{0}+: \phi_{1} \phi_{3}:\left\langle\phi_{2} \phi_{4}\right\rangle_{0} \\
+: \phi_{1} \phi_{4}:\left\langle\phi_{2} \phi_{3}\right\rangle_{0}+: \phi_{2} \phi_{3}:\left\langle\phi_{1} \phi_{4}\right\rangle_{0}+: \phi_{2} \phi_{4}:\left\langle\phi_{1} \phi_{3}\right\rangle_{0} \\
+: \phi_{3} \phi_{4}:\left\langle\phi_{1} \phi_{2}\right\rangle_{0}+\left\langle\phi_{1} \phi_{2}\right\rangle_{0}\left\langle\phi_{3} \phi_{4}\right\rangle_{0}+\left\langle\phi_{1} \phi_{3}\right\rangle_{0}\left\langle\phi_{2} \phi_{4}\right\rangle_{0} \\
\quad+\left\langle\phi_{1} \phi_{4}\right\rangle_{0}\left\langle\phi_{2} \phi_{3}\right\rangle_{0},
\end{aligned}
$$

where $\langle\cdots\rangle_{0}$ denotes the expectation value of the scalar field in the Minkowski vacuum. The first term is a fully normal-ordered term, the next six terms are cross terms, and the final three terms are pure vacuum terms. For a single-mode coherent state, the fully normalordered terms cancel, and then we are left with

$$
\begin{aligned}
\left\langle\phi_{1} \phi_{2} \phi_{3} \phi_{4}\right\rangle_{\bar{\alpha}}-\left\langle\phi_{1} \phi_{2}\right\rangle_{\bar{\alpha}}\left\langle\phi_{3} \phi_{4}\right\rangle_{\bar{\alpha}}=\langle: & \left.\phi_{1} \phi_{3}:\right\rangle_{\bar{\alpha}}\left\langle\phi_{2} \phi_{4}\right\rangle_{0}+\text { other cross terms } \\
& +\left\langle\phi_{1} \phi_{2}\right\rangle_{0}\left\langle\phi_{3} \phi_{4}\right\rangle_{0}+\text { other pure vacuum terms } .
\end{aligned}
$$

The cross terms and the pure vacuum terms may be singular because the field operators will be evaluated at the same point. It has been shown that the spacetime average of the pure vacuum term leads to a result that varies as an inverse power of the size of the spacetime [17]. On contrary, the cross terms depend on the particle density of the coherent state. For a large value of the particle density, the cross terms can dominate over the pure vacuum terms [17. Thus we will consider the contributions from the cross terms and ignore the pure vacuum terms. Since the integrals that contain cross terms may still diverge, a finite result is obtained [17] by choosing the principal value of the singular integral. Here we take an alternative approach to cope with the singular vacuum contribution so that all results become regular. They prove to be the same in the end. 
It is straightforward to compute the expectation value of the operators in Eq. (16). Let us focus on the large $t \gg L-z_{0}$ limit. They are given by

$$
\langle q(t)\rangle_{\bar{\alpha}}=|\bar{\alpha}|^{2} \frac{\mathcal{A}_{\|}}{(2 \pi)^{3}} \frac{\bar{\omega}}{m} \times t^{2} .
$$

The results of $\left\langle\partial_{L} I\right\rangle_{\bar{\alpha}}$ and $\left\langle\partial_{L}^{2} I\right\rangle_{\bar{\alpha}}$ can be obtained by taking the derivative of the expression Eq. 15) with respect to $L$.

Let the dispersion associated with $\Delta z$ be defined as

$$
\left\langle\Delta z^{2}\right\rangle=\left\langle(\Delta z)^{2}\right\rangle=\frac{\left\langle\Delta I^{2}\left(z_{0}, t ; q+L\right)\right\rangle_{\bar{\alpha}}}{\left\langle\partial_{L} I_{T}\left(z_{0}, t ; L\right)\right\rangle_{\bar{\alpha}}^{2}} .
$$

From (16), the whole expressions in $\left\langle\Delta z^{2}\right\rangle$ can be grouped according to their physical meaning. They respectively come from the shot noise (sn) contribution associated with intrinsic fluctuations of the incident coherent fields, and the contributios of radiation pressure fluctuations (rp) and modified field fluctuations (mf), both of which are induced by the mirror's motion,

$$
\begin{aligned}
\left\langle\Delta z^{2}\right\rangle_{s n} & =\frac{\left\langle\Delta I^{2}\left(z_{0}, t ; L\right)\right\rangle_{\bar{\alpha}}}{\left\langle\partial_{L} I\left(z_{0}, t ; L\right)\right\rangle_{\bar{\alpha}}^{2}} \\
\left\langle\Delta z^{2}\right\rangle_{r p} & =\left\langle\Delta q^{2}(t)\right\rangle_{\bar{\alpha}} \\
\left\langle\Delta z^{2}\right\rangle_{m f} & =\langle q(t)\rangle_{\bar{\alpha}}^{2} \frac{\left\langle\Delta\left(\partial_{L} I\right)^{2}\left(z_{0}, t ; L\right)\right\rangle_{\bar{\alpha}}}{\left\langle\partial_{L} I\left(z_{0}, t ; L\right)\right\rangle_{\bar{\alpha}}^{2}} .
\end{aligned}
$$

In addition and more importantly, there exist the cross terms owing to correlation between different sources of uncertainty. Up to the order $q^{2}$, we lump these terms together in

$$
\begin{aligned}
\left\langle\Delta z^{2}\right\rangle_{\text {cor }}= & \frac{1}{\left\langle\partial_{L} I(t)\right\rangle_{\bar{\alpha}}}\langle\{\Delta I(t), \Delta q(t)\}\rangle_{\bar{\alpha}}+\frac{\langle q(t)\rangle_{\bar{\alpha}}}{\left\langle\partial_{L} I(t)\right\rangle_{\bar{\alpha}}^{2}}\left\langle\left\{\Delta I(t), \Delta \partial_{L} I(t)\right\}\right\rangle_{\bar{\alpha}} \\
& +\frac{\langle q(t)\rangle_{\bar{\alpha}}}{\left\langle\partial_{L} I(t)\right\rangle_{\bar{\alpha}}}\left\langle\left\{\Delta \partial_{L} I(t), \Delta q(t)\right\}\right\rangle_{\bar{\alpha}}+\frac{\langle q(t)\rangle_{\bar{\alpha}}\left\langle\partial_{L}^{2} I(t)\right\rangle_{\bar{\alpha}}}{\left\langle\partial_{L} I(t)\right\rangle_{\bar{\alpha}}^{2}}\langle\{\Delta I(t), \Delta q(t)\}\rangle_{\bar{\alpha}} \\
& +\frac{1}{2} \frac{\langle q(t)\rangle_{\bar{\alpha}}^{2}}{\left\langle\partial_{L} I(t)\right\rangle_{\bar{\alpha}}^{2}}\left\langle\left\{\Delta \partial_{L}^{2} I(t), \Delta I(t)\right\}\right\rangle_{\bar{\alpha}} .
\end{aligned}
$$

From now afterwards, the quantity $I$ and its derivatives are understood to be evaluated at $z=L$, and we replace $I\left(z_{0}, t ; L\right)$ simply by its shorthand notation $I(t)$. We will discuss these contributions individually in the following sections. In particular, the emphasis will be put on the correlation effects that may give the hope to reduce the overall uncertainty. Here we note that Eqs (21), (22), (23), (24) involve the expectation values of the anticommutators of the operators, so in the leading-order approximation we will obtain the same results irrespective of operator ordering on expanding Eq. (11). 


\section{A. shot-noise terms}

To the overall dispersion of $\Delta z$, the contribution from the intrinsic fluctuations of the incident field is given by the term $\left\langle\Delta I^{2}\right\rangle_{\bar{\alpha}}$, which is expressed as

$$
\begin{aligned}
& \left\langle\Delta I^{2}\left(z_{0}, t ; L\right)\right\rangle_{\bar{\alpha}}=\left\langle I^{2}\left(z_{0}, t ; L\right)\right\rangle_{\bar{\alpha}}-\left\langle I\left(z_{0}, t ; L\right)\right\rangle_{\bar{\alpha}}^{2} \\
& =\int_{0}^{t} d s \int d \mathcal{A}_{\|} \int^{\prime} \frac{d^{3} k}{(2 \pi)^{\frac{3}{2}}} \int^{\prime} \frac{d^{3} k^{\prime}}{(2 \pi)^{\frac{3}{2}}} \int_{0}^{t} d s^{\prime} \int d \mathcal{A}_{\|}^{\prime} \int^{\prime} \frac{d^{3} \tilde{k}}{(2 \pi)^{\frac{3}{2}}} \int^{\prime} \frac{d^{3} \tilde{k}^{\prime}}{(2 \pi)^{\frac{3}{2}}} \frac{1}{\sqrt{2 k}} \frac{1}{\sqrt{2 k^{\prime}}} \frac{1}{\sqrt{2 \tilde{k}}} \frac{1}{\sqrt{2 \tilde{k}^{\prime}}} \\
& \times \mathcal{U}_{\mathbf{k}}^{*}\left(\mathbf{x}_{\|}, z_{0}, s ; L\right) \mathcal{U}_{\mathbf{k}^{\prime}}\left(\mathbf{x}_{\|}, z_{0}, s ; L\right) \mathcal{U}_{\hat{\mathbf{k}}}^{*}\left(\mathbf{x}_{\|}^{\prime}, z_{0},, s^{\prime} ; L\right) \mathcal{U}_{\tilde{\mathbf{k}}^{\prime}}\left(\mathbf{x}_{\|}^{\prime}, z_{0}, s^{\prime} ; L\right) \\
& \times\left[\left\langle a_{\mathbf{k}}^{\dagger} a_{\mathbf{k}^{\prime}} a_{\tilde{\mathbf{k}}}^{\dagger} a_{\tilde{\mathbf{k}}^{\prime}}\right\rangle_{\bar{\alpha}}-\left\langle a_{\mathbf{k}}^{\dagger} a_{\mathbf{k}^{\prime}}\right\rangle_{\bar{\alpha}}\left\langle a_{\tilde{\mathbf{k}}}^{\dagger} a_{\tilde{\mathbf{k}}^{\prime}}\right\rangle_{\bar{\alpha}}\right] \\
& =\frac{\mathcal{A}_{\|}}{(2 \pi)^{3}}|\bar{\alpha}|^{2} \frac{8}{\bar{\omega}} \sin ^{2}\left[\bar{\omega}\left(L-z_{0}\right)\right] \int_{0}^{t} d s \int_{0}^{t} d s^{\prime} \int_{0}^{\infty} \frac{d \omega}{2 \pi} \frac{1}{2 \omega} \sin ^{2}\left[\omega\left(L-z_{0}\right)\right] e^{-i(\omega-\bar{\omega}) s} e^{i(\omega-\bar{\omega}) s^{\prime}},
\end{aligned}
$$

where the area integral is carried out over a measuring plane at $z=z_{0}$, and its total area $\mathcal{A}_{\|}$ is assume to be large but finite. Performing the time integrations and using the fact that

$$
\lim _{t \rightarrow \infty} \frac{1}{\omega-\bar{\omega}} \sin \left[\frac{(\omega-\bar{\omega}) t}{2}\right]=\pi \delta\left(\omega-\omega_{0}\right)
$$

we have

$$
\left\langle\Delta I^{2}\left(z_{0}, t ; L\right)\right\rangle_{\bar{\alpha}} \approx \frac{\mathcal{A}_{\|}}{(2 \pi)^{3}}|\bar{\alpha}|^{2} \frac{4}{\bar{\omega}^{2}} \sin ^{4}\left[\bar{\omega}\left(L-z_{0}\right)\right] \times t .
$$

Together with the normalization factor $\left\langle\partial_{L} I\right\rangle_{\bar{\alpha}}^{2}$, Eq. 21) gives the positive contribution

$$
\left\langle\Delta z^{2}\right\rangle_{s n}=\frac{\left\langle\Delta I^{2}\right\rangle_{\bar{\alpha}}}{\left\langle\partial_{L} I\right\rangle_{\bar{\alpha}}^{2}} \approx \frac{1}{P \bar{\omega} t} \frac{1}{4} \tan ^{2}\left[\bar{\omega}\left(L-z_{0}\right)\right], \quad t \gg 1 / \bar{\omega}, \quad \text { and } \quad t \gg L-z_{0},
$$

where the energy flux $P$ of the incident state is defined by

$$
P=\frac{\mathcal{A}_{\|}}{(2 \pi)^{3}}|\bar{\alpha}|^{2} \bar{\omega} .
$$

The result (27) is inversely proportional to the power $P$, and that is the typical behavior of the shot nose.

Since the read-out field $I\left(z_{0}, t ; L\right)$ is to count the number of the particles being detected, the same result can be obtained in terms of the particle number operator $n$ if we make such an identification,

$$
I\left(z_{0}, t ; L\right)=\frac{2}{\bar{\omega}} \sin ^{2}\left[\bar{\omega}\left(L-z_{0}\right)\right] \times n,
$$

and notice that for a coherent state, $\left\langle n^{2}\right\rangle_{\bar{\alpha}}-\langle n\rangle_{\bar{\alpha}}^{2}=\langle n\rangle_{\bar{\alpha}}$, where the mean number of particles $\langle n\rangle_{\bar{\alpha}}$ that reaches the surface $\mathcal{A}_{\|}$within time $t$ is that $\langle n\rangle_{\bar{\alpha}}=\frac{\mathcal{A}_{\|}}{(2 \pi)^{3}}|\bar{\alpha}|^{2} t$. This shot noise can be understood as phase fluctuations of the field [26]. The phase uncertainty of $I$ can be effectively defined as

$$
\Delta \phi=\frac{\sqrt{\left\langle\Delta^{2} I(\phi, t)\right\rangle_{\bar{\alpha}}}}{\left|\left\langle\partial_{\phi} I(\phi, t)\right\rangle_{\bar{\alpha}}\right|}
$$


The variance of the phase $\Delta \phi$ is found proportional to $1 / P$ as well. It is worth to mention that this result depends on not only the input power $P$ but also the distance between the mirror and detector due to interference between the incident and reflected fields. The proper choice of the parameter $L-z_{0}$ can be used to avoid the potentially large uncertainty due to the factor $\tan ^{2}\left[\bar{\omega}\left(L-z_{0}\right)\right]$ and then to minimize the overall uncertainty. The same strategy has been used by Caves in [3, 4] to finding the appropriate spot in the fringe pattern so as to further reduce the overall uncertainty in the interferometer.

\section{B. radiation-pressure-fluctuations terms}

Next, we consider the contribution to $\left\langle\Delta z^{2}\right\rangle$ from the radiation pressure fluctuations. This contribution is given by $\left\langle\Delta q^{2}(t)\right\rangle_{\bar{\alpha}}$ :

$$
\left\langle\Delta z^{2}\right\rangle_{r p}=\left\langle\Delta q^{2}(t)\right\rangle_{\bar{\alpha}}=\left\langle q^{2}\right\rangle_{\bar{\alpha}}-\langle q\rangle_{\bar{\alpha}}^{2}
$$

where the most dominant contribution is found to be

$$
\begin{aligned}
& \left\langle\Delta q^{2}\right\rangle_{\bar{\alpha}}=\int_{0}^{t} d \tau \int_{0}^{\tau} d s \int d \mathcal{A}_{\|} \int_{0}^{t} d \tau^{\prime} \int_{0}^{\tau^{\prime}} d s^{\prime} \int d \mathcal{A}_{\|}^{\prime} \\
& \int^{\prime} \frac{d^{3} k}{(2 \pi)^{\frac{3}{2}}} \int^{\prime} \frac{d^{3} k^{\prime}}{(2 \pi)^{\frac{3}{2}}} \int^{\prime} \frac{d^{3} \tilde{k}}{(2 \pi)^{\frac{3}{2}}} \int^{\prime} \frac{d^{3} \tilde{k}^{\prime}}{(2 \pi)^{\frac{3}{2}} \sqrt{2 k}} \frac{1}{\sqrt{2 k^{\prime}}} \frac{1}{\sqrt{2 \tilde{k}}} \frac{1}{\sqrt{2 \tilde{k}^{\prime}}} \\
& \left\{\partial_{z_{0}} \mathcal{U}_{\mathbf{k}}^{*}\left(\mathbf{x}_{\|}, z_{0}, s\right) \partial_{z_{0}} \mathcal{U}_{\mathbf{k}^{\prime}}\left(\mathbf{x}_{\|}, z_{0}, s\right) \partial_{z_{0}} \mathcal{U}_{\tilde{\mathbf{k}}}^{*}\left(\tilde{\mathbf{x}}_{\|}, z_{0}, s^{\prime}\right) \partial_{z_{0}} \mathcal{U}_{\tilde{\mathbf{k}}^{\prime}}\left(\tilde{\mathbf{x}}_{\|}, z_{0}, s^{\prime}\right)\right. \\
& \times\left[\left\langle a_{k}^{\dagger} a_{k^{\prime}} a_{\tilde{k}}^{\dagger} a_{\tilde{k}^{\prime}}\right\rangle_{\bar{\alpha}}-\left\langle a_{k}^{\dagger} a_{k^{\prime}}\right\rangle_{\bar{\alpha}}\left\langle a_{\tilde{k}}^{\dagger} a_{\tilde{k}^{\prime}}\right\rangle_{\bar{\alpha}}\right] \\
& +\partial_{z_{0}} \mathcal{U}_{\mathbf{k}^{\prime}}^{*}\left(\mathbf{x}_{\|}, z_{0}, s\right) \partial_{z_{0}} \mathcal{U}_{\mathbf{k}}\left(\mathbf{x}_{\|}, z_{0}, s\right) \partial_{z_{0}} \mathcal{U}_{\tilde{\mathbf{k}}^{\prime}}^{*}\left(\tilde{\mathbf{x}}_{\|}, z_{0}, s^{\prime}\right) \partial_{z_{0}} \mathcal{U}_{\tilde{\mathbf{k}}}\left(\tilde{\mathbf{x}}_{\|}, z_{0}, s^{\prime}\right) \\
& \left.\times\left[\left\langle a_{k^{\prime}}^{\dagger} a_{k} a_{\tilde{k}^{\prime}}^{\dagger} a_{\tilde{k}}\right\rangle_{\bar{\alpha}}-\left\langle a_{k^{\prime}}^{\dagger} a_{k}\right\rangle_{\bar{\alpha}}\left\langle a_{\tilde{k}^{\prime}}^{\dagger} a_{\tilde{k}}\right\rangle_{\bar{\alpha}}\right]+\text { c.c. }\right\}_{z_{0}=L}+\text { subdominant terms } \\
& =\frac{4 \bar{\omega}}{m^{2}} \frac{\mathcal{A}_{\|}}{(2 \pi)^{3}}|\bar{\alpha}|^{2} \int_{0}^{t} d \tau \int_{0}^{\tau} d s \int_{0}^{t} d \tau^{\prime} \int_{0}^{\tau^{\prime}} d s^{\prime} \int_{0}^{\infty} \frac{d \omega}{2 \pi} \omega e^{-i(\omega-\bar{\omega}) s} e^{i(\omega-\bar{\omega}) s^{\prime}} \\
& + \text { subdominant terms } \\
& \approx \frac{|\bar{\alpha}|^{2} \bar{\omega}}{m^{2}} \frac{\mathcal{A}_{\|} t^{2}}{(2 \pi)^{3}} \bar{\omega} t=\frac{P \bar{\omega} t^{3}}{m^{2}}
\end{aligned}
$$

This result is valid for large time. All other subdominant contributions will be discussed in Appendix $\mathrm{B}$. The result of $\left\langle\Delta q^{2}\right\rangle_{\bar{\alpha}}$ at large time can be equivalently obtained by the particle-number operator approach, and it is given by the time integration of the velocity operator of the form

$$
v=\frac{2 \bar{\omega}}{m} n,
$$


where $n$ is the particle number operator. In other words, the momentum received by the mirror can be effectively given by the momentum transfer of the scalar particles when they elastically bounce off the mirror.

In contrast to the shot-noise type contribution that has the $P^{-1}$ dependence, the stochastic motion of the mirror due to radiation pressure fluctuations yields an uncertainty proportional to the incident power $P$. This uncertainty can be attributed to the amplitude fluctuations of the incident field. These two types of noise have been extensively discussed in [3, 4, 7] in the context of the interferometer. In particular, it is stated in [10] that shot noise given by the photon counting error can be understood in terms of phase fluctuations of the incident light while radiation pressure fluctuations are related to amplitude fluctuations. Our results are consistent with the arguments in [3, 4, 7, 10].

Other than contributions related to well-known shot noise and radiation-pressure fluctuations, modified field fluctuations also brings in a new component of noise. As the radiation pressure pushes the mirror into motion, the scalar field in the proximity of the mirror must change accordingly at every moment to enforce the boundary condition. In turn, this modification of field fluctuations will manifest themselves as quantum noise. Since these fluctuations arise naturally from the moving boundary condition of the fields, it must be included consistently in the expansion of Eq. (16), and this adds a new contribution into $\Delta z^{2}$, as is described by (23). With the help of Eq. 19p and

$$
\left\langle\Delta\left(\partial_{L} I\right)^{2}\left(z_{0}, t ; L\right)\right\rangle_{\bar{\alpha}}=\left\langle\left(\partial_{L} I\right)^{2}\right\rangle_{\bar{\alpha}}-\left\langle\partial_{L} I\right\rangle_{\bar{\alpha}}^{2} \approx 4 \frac{\mathcal{A}_{\|}}{(2 \pi)^{3}}|\bar{\alpha}|^{2} \sin ^{2}\left[2 \bar{\omega}\left(L-z_{0}\right)\right] \times t,
$$

we have

$$
\left\langle\Delta z^{2}\right\rangle_{m f}=\frac{\langle q\rangle_{\bar{\alpha}}^{2}}{\left\langle\partial_{L} I\right\rangle_{\bar{\alpha}}^{2}}\left\langle\Delta^{2} \partial_{L} I\right\rangle_{\bar{\alpha}} \approx \frac{P \bar{\omega} t^{3}}{m^{2}},
$$

for $\bar{\omega} t \gg 1$. Apparently, this term has mixed features that involve $\langle q\rangle_{\alpha}$ and $\left\langle\partial_{L} I\right\rangle_{\bar{\alpha}}$. Hence it may depend on both the incident power and the phase of incident state. In the end, however, the phase dependence cancels in this configuration so that the noise from modified field fluctuations depends only on the power of the input state.

\section{C. correlation terms}

In this framework, it is quite straightforward to compute the correlation between various noise. The correlation between shot noise and noise from random motion is given by

$$
\frac{1}{\left\langle\partial_{L} I\right\rangle_{\bar{\alpha}}}\langle\{\Delta I, \Delta q\}\rangle_{\bar{\alpha}} \approx \frac{t}{m} \tan \left[\bar{\omega}\left(L-z_{0}\right)\right],
$$

which can take either sign, depending on the distance between the detector and the mirror's original position. The presence of such sign-vary expressions gives the hope to reduce the 
overall uncertainty $\left\langle\Delta z^{2}\right\rangle_{\bar{\alpha}}$. It is worth to mention that the same result can be obtained by the particle-number operator approach using Eqs. (29), and (33).

There exists another correlation term due to the additional source of noise, that is, the correlation between shot noise and the noise due to modified field fluctuation,

$$
\frac{\langle q\rangle_{\bar{\alpha}}}{\left\langle\partial_{L} I\right\rangle_{\bar{\alpha}}^{2}}\left\langle\left\{\Delta I, \Delta \partial_{L} I\right\}\right\rangle_{\bar{\alpha}} \approx \frac{t}{m} \tan \left[\bar{\omega}\left(L-z_{0}\right)\right],
$$

which has the same order of the magnitude as the the previous result. Furthermore, it leads to

$$
\begin{aligned}
& \frac{\langle q\rangle_{\bar{\alpha}}}{\left\langle\partial_{L} I\right\rangle_{\bar{\alpha}}}\left\langle\left\{\Delta \partial_{L} I, \Delta q\right\}\right\rangle_{\bar{\alpha}}+\frac{\langle q\rangle_{\bar{\alpha}}\left\langle\partial_{L}^{2} I\right\rangle_{\bar{\alpha}}}{\left\langle\partial_{L} I\right\rangle_{\bar{\alpha}}^{2}}\langle\{\Delta I, \Delta q\}\rangle_{\bar{\alpha}}+\frac{1}{2} \frac{\langle q\rangle_{\bar{\alpha}}^{2}}{\left\langle\partial_{L} I\right\rangle_{\bar{\alpha}}^{2}}\left\langle\left\{\Delta \partial_{L}^{2} I, \Delta I\right\}\right\rangle_{\bar{\alpha}} \\
& \approx \frac{P \bar{\omega} t^{3}}{m^{2}}\left(\frac{7}{2}-\frac{3}{2} \tan ^{2}\left[\bar{\omega}\left(L-z_{0}\right)\right]\right), \quad \quad \bar{\omega} t \gg 1,
\end{aligned}
$$

when we add up correlation terms of the order $q^{2}$, as is summarized within the expression $\left\langle\Delta^{2} z\right\rangle_{\text {cor }}$ in Eq. (24).

Now we may put all effects together, and the overall uncertainty $\left\langle\Delta z^{2}\right\rangle_{\bar{\alpha}}$ is then given by

$$
\left\langle\Delta z^{2}\right\rangle_{\bar{\alpha}}=\frac{1}{P \bar{\omega} t} \frac{\zeta^{2}}{4}+\frac{t}{m} 2 \zeta+\frac{P \bar{\omega} t^{3}}{m^{2}}\left(\frac{11}{2}-\frac{3}{2} \zeta^{2}\right)
$$

where $\zeta=\tan \left[\bar{\omega}\left(L-z_{0}\right)\right]$. Interference between the incoming and reflected waves gives the quantity $I$ the dependence on the effective distance between the mirror and detector. Later, the correlation between different sources of quantum noise can be found to possibly reduced the overall quantum noise.

\section{EFFECT OF CORRELATION}

In the case of single-mode coherent state, $\zeta>0$ represents positive correlation between shot noise and radiation-pressure fluctuations, while $\zeta<0$ denotes negative correlation. The minimum value of $\left\langle\Delta z^{2}\right\rangle_{\bar{\alpha}}$ is found to be

$$
\min \left\langle\Delta z^{2}\right\rangle_{\bar{\alpha}}=\zeta\left(2 \pm \sqrt{\frac{11}{2}-\frac{3}{2} \zeta^{2}}\right) \frac{t}{m},
$$

with an optimal value of the power $P$ given by

$$
P_{\mathrm{opt}}= \pm \frac{\zeta}{\sqrt{22-6 \zeta^{2}}} \frac{m}{\bar{\omega} t^{2}} .
$$

In Eqs. (36) and (37), the "+ (-)" sign corresponds to the positive (negative) $\zeta$ case.

The minimal value of $\left\langle\Delta z^{2}\right\rangle_{\bar{\alpha}}$ depends on $\zeta$, but the range of the values of $\zeta$ can not be arbitrary; instead, it has to be determined in a consistent way with the underlying 


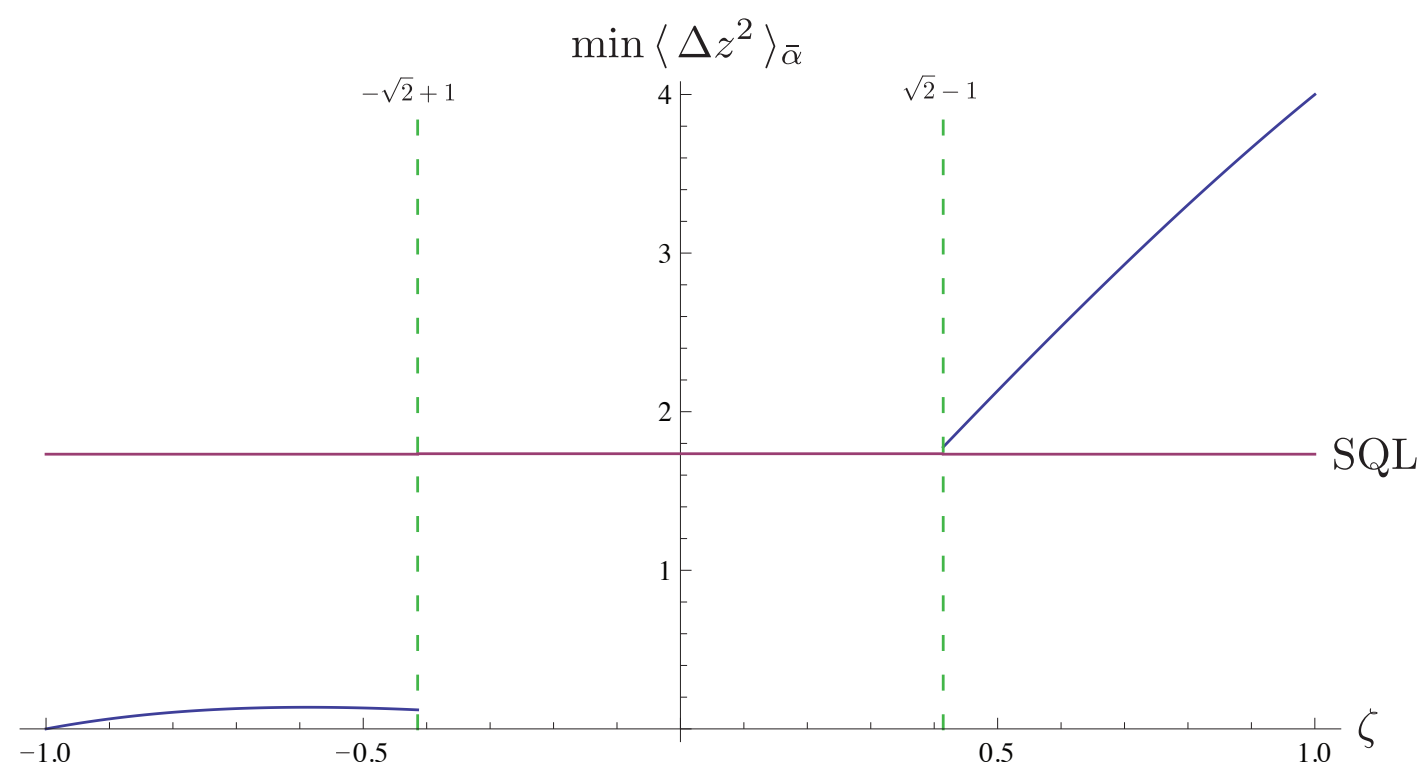

FIG. 2: The minimal value of $\left\langle\Delta z^{2}\right\rangle_{\bar{\alpha}}$ as a function of $\zeta$ in comparison with the SQL. The $\min \left\langle\Delta z^{2}\right\rangle_{\bar{\alpha}}$ is drawn in the unit of $\frac{t}{m}$.

assumptions. Slow motion of the mirror, $\bar{\omega} q \ll 1$, can be translated into an inequality, $P_{\text {opt }} \bar{\omega} t^{2} / m \ll 1$, as can be seen from (19) and (28). The latter in turn implies $|\zeta|<1$ by substituting the expression of $P_{\mathrm{opt}}$ in (37) directly into the inequality. On the other hand, the definition of the effective displacement of the mirror in $(17)$, formally by $\Delta I \approx \Delta z \times \partial_{L} I$, implicitly assumes

$$
\left|\Delta z \times \partial_{L} I\right|>\left|\frac{1}{2}(\Delta z)^{2} \partial_{L}^{2} I\right|
$$

Together with the slow motion assumption, it gives

$$
|\bar{\omega} \Delta z|<\frac{2|\zeta|}{1-\zeta^{2}}<1
$$

The last inequality is imposed to ensure that the range of the value of $\Delta z$ is consistent with nonrelativistic motion of the mirror as well as the requirement on the definition of $\Delta z$ in (17), leading to $|\zeta|>\sqrt{2}-1 \sim 0.414$. Thus $|\zeta|$ should lie within the range

$$
\sqrt{2}-1<|\zeta|<1
$$

The minimal value of $\left\langle\Delta z^{2}\right\rangle_{\bar{\alpha}}$ as a function of $\zeta$ is shown in Fig. 2 .

For a positive $\zeta, \min \left\langle\Delta z^{2}\right\rangle_{\bar{\alpha}}$ is a monotonic function of $\zeta$, so it varies between

$$
1.78 \frac{t}{m}<\min \left\langle\Delta z^{2}\right\rangle_{\bar{\alpha}}<4 \frac{t}{m},
$$

compared with the SQL, $\Delta z_{\mathrm{SQL}}^{2}=t / m$. It is seen that we cannot beat $\mathrm{SQL}$ in the positive correlation case. For the upper (lower) bound in (38), the corresponding optimal power is 
given by

$$
P_{\mathrm{opt}} \approx 0.25 \frac{m}{\bar{\omega} t^{2}} \quad \text { and } \quad P_{\mathrm{opt}} \approx 0.09 \frac{\mathrm{m}}{\bar{\omega} t^{2}},
$$

respectively. On the other hand, for negative $\zeta, \min \left\langle\Delta^{2} z\right\rangle_{\bar{\alpha}}$ is not a monotonic with $\zeta$, having a maximum at $\zeta=-0.588$, so in this case the largest value of $\min \left\langle\Delta^{2} z\right\rangle_{\bar{\alpha}}$ is

$$
\min \left\langle\Delta z^{2}\right\rangle_{\bar{\alpha}} \approx 0.136 \frac{t}{m}
$$

with the optimal input power

$$
P_{\mathrm{opt}} \approx 0.132 \frac{m}{\bar{\omega} t^{2}} .
$$

In the case of negative correlation, even the largest value of $\min \left\langle\Delta^{2} z\right\rangle_{\bar{\alpha}}$ can be lower than the standard quantum limit. Therefore, the value of the position uncertainty can be further reduced by other choices of $\zeta$, and naïvely it can be as small as possible. However, there is a caveat. We have ignored higher-order terms in the series expansion (13) and in the calculation of $\left\langle\Delta z^{2}\right\rangle_{\bar{\alpha}}$ in terms of small $q$. In addition, we have employed the leading order approximation in the long-time limit by considering a large number of the particles in the coherent state. Hence $\min \left\langle\Delta z^{2}\right\rangle_{\bar{\alpha}}$ cannot be arbitrarily small in that the subleading results will sooner or later contribute comparable effects. At any rate, we have shown that the standard quantum limit can be possibly overcome by establishing correlation between the intrinsic fluctuations of the field (shot noise) and its induced fluctuations due to the dynamics of the mirror (radiation pressure fluctuations and modified field fluctuations).

So far, we have considered the single-mode coherent state. In reality, coherent state is at best prepared with a finite frequency bandwidth, so the observed response of the detector should be averaged over its bandwidth. In general, let the frequency distribution be described by some function of the form $f\left(\omega, \bar{\omega} ; \sigma_{0}\right)$, where $\bar{\omega}$ is the mean frequency of the band and $2 \sigma_{0}$ the bandwidth. The average over the distribution function is given by

$$
\bar{O}\left(\bar{\omega}, \sigma_{0}\right)=\int_{0}^{\infty} d \omega f\left(\omega, \bar{\omega} ; \sigma_{0}\right) O(\omega) .
$$

In our case, the quantity $O$ is a product of a fast oscillating function of $\omega$ due to the macroscopic scale $L-z_{0}$ and a relatively slowly varying component over the interval $\bar{\omega}-\sigma_{0}<$ $\omega<\bar{\omega}+\sigma_{0}$. Suppose the bandwith is narrow in the sense that $\sigma_{0} \ll \bar{\omega}$ and $\sigma_{0}\left(L-z_{0}\right) \gg$ 1. Then the average can be approximated by substituting the variable $\omega$ in the slowly varying component with the mean frequency $\bar{\omega}$ and directly performing average over the fast oscillating part.

Thus if the coherent parameter $\alpha$ is independent of the frequency, the average of the normalization factor $\left\langle\partial_{L} I\right\rangle_{\bar{\alpha}}^{2}$ is given by

$$
\overline{\left\langle\partial_{L} I\right\rangle_{\bar{\alpha}}^{2}}=4|\bar{\alpha}|^{4} \frac{\mathcal{A}_{\|}^{2}}{(2 \pi)^{6}} \overline{\sin ^{2}\left[2 \omega\left(L-z_{0}\right)\right]} \times t^{2} \approx 2|\bar{\alpha}|^{4} \frac{\mathcal{A}_{\|}^{2}}{(2 \pi)^{6}} \times t^{2},
$$


from Eq. 15]. Likewise, the corresponding frequency averages of the various sources of noise can be computed. The average of the shot noise term is given by

$$
{\overline{\left\langle\Delta I^{2}\right\rangle_{s n}}}=4|\bar{\alpha}|^{2} \frac{\mathcal{A}_{\|}}{(2 \pi)^{3}} \frac{1}{\bar{\omega}^{2}} \overline{\sin ^{4}\left[\omega\left(L-z_{0}\right)\right]} \times t \approx|\bar{\alpha}|^{2} \frac{\mathcal{A}_{\|}}{(2 \pi)^{3}} \frac{3}{2 \bar{\omega}^{2}} \times t,
$$

and the average of the radiation-pressure fluctuations contribution is

$$
{\overline{\left\langle\Delta I^{2}\right\rangle_{r p}}}=|\bar{\alpha}|^{6} \frac{\mathcal{A}_{\|}^{3}}{(2 \pi)^{9}} \frac{16 \bar{\omega}^{2}}{m^{2}} \overline{\sin ^{2}\left[\omega\left(L-z_{0}\right)\right] \cos ^{2}\left[\omega\left(L-z_{0}\right)\right]} \times t^{5} \approx|\bar{\alpha}|^{6} \frac{\mathcal{A}_{\|}^{3}}{(2 \pi)^{9}} \frac{2 \bar{\omega}^{2}}{m^{2}} \times t^{5} .
$$

It is interesting to note that the average of the rest of the terms totally cancels out by themselves, even though they contain drastically different information about correlation between noises and modified field fluctuations,

$$
{\overline{\left\langle\Delta I^{2}\right\rangle_{m f}}}+{\overline{\left\langle\Delta I^{2}\right\rangle_{c o r}}}=0
$$

The presence of finite frequency bandwidth reduces $\left\langle\Delta I^{2}\left(z_{0}, t ; q+L\right)\right\rangle$ to solely consist of contributions of shot noise and radiation-pressure fluctuations. Hence $\overline{\left\langle\Delta z^{2}\right\rangle_{\bar{\alpha}}}$ in the end is given by

$$
\overline{\left\langle\Delta z^{2}\right\rangle_{\bar{\alpha}}}=\frac{\overline{\left\langle\Delta I^{2}\right\rangle_{\bar{\alpha}}}}{\overline{\left\langle\partial_{L} I\right\rangle_{\bar{\alpha}}^{2}}}=\frac{3}{4} \frac{1}{P \bar{\omega} t}+\frac{P \bar{\omega} t^{3}}{m^{2}},
$$

which can be compared with Eq. (35). Minimization of $\overline{\left\langle\Delta z^{2}\right\rangle_{\bar{\alpha}}}$ can be achieved with an optimal value of the power $P$,

$$
P_{\mathrm{opt}}=\frac{\sqrt{3}}{2} \frac{m}{\bar{\omega} t^{2}}
$$

leading to the minimal value of $\overline{\left\langle\Delta z^{2}\right\rangle_{\bar{\alpha}}}$,

$$
\min \overline{\left\langle\Delta z^{2}\right\rangle_{\bar{\alpha}}}=\sqrt{3} \frac{t}{m} .
$$

The comparison will be made clear in Fig. 3 to highlight the significance of the correlation effects.

At last we would like to make some remarks about the standard quantum limit in some other often-studied examples in the context of the measurement of the position of a quantummechanically free mass [2]. In those examples, the basic idea is that the evolution of the position of a free mass at time $t$ is given by $q(t)=q(0)+p(0) t / m$. Thus, its position fluctuations with respect to a prescribed quantum state then turn out to be

$$
\left\langle\Delta q^{2}(t)\right\rangle=\left\langle\Delta q^{2}(0)\right\rangle+\left\langle\Delta p^{2}(0)\right\rangle \frac{t^{2}}{m^{2}}+\langle\Delta q(0) \Delta p(0)+\Delta p(0) \Delta q(0)\rangle \frac{t}{m} .
$$

It is assumed that the correlation term in the last expression is either vanishing or positive. The minimum position fluctuations is achieved by considering the zero correlation. Then 

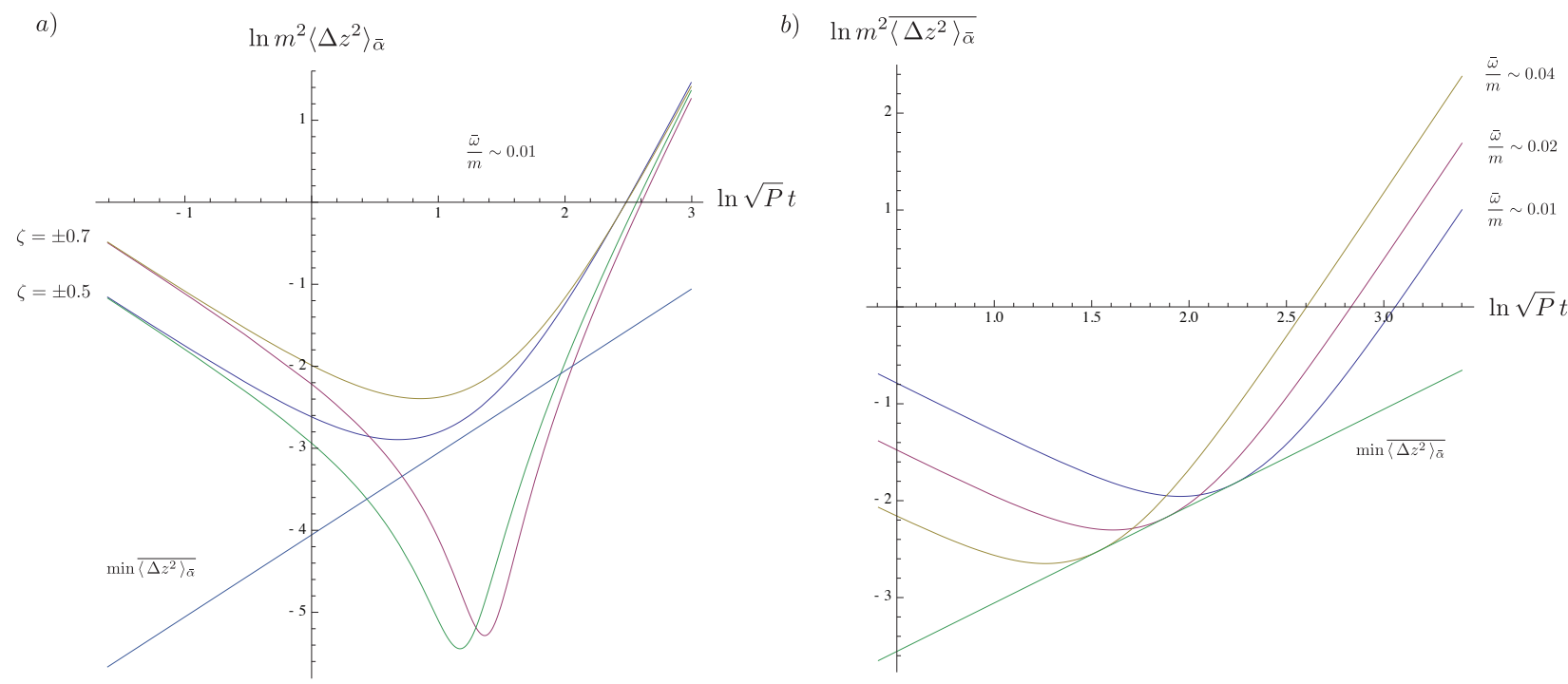

FIG. 3: a) Log-log plot of $\left\langle\Delta z^{2}\right\rangle_{\bar{\alpha}}$ versus $\sqrt{P} t$ for various values of $\zeta$. The straight line corresponds to the SQL. The parameters $\frac{\bar{\omega}}{m}=10^{-2}$ and $\frac{P}{m^{2}}=10^{-4}$ are chosen. b) Log-log plot of $\overline{\left\langle\Delta z^{2}\right\rangle_{\bar{\alpha}}}$ versus $\sqrt{P} t$ for various values of $\frac{\bar{\omega}}{m}$. The straight line is the result of $\min \overline{\left\langle\Delta z^{2}\right\rangle_{\bar{\alpha}}} \cdot \frac{P}{m^{2}}=10^{-4}$ is chosen.

by applying the minimum position-momentum uncertainty and minimizing the remaining expressions, we obtain the SQL. However, in [5], Yuen points out that the correlation term surely depends on the quantum state under consideration, and can be negative in principle. Thus, in general, beating the SQL is viable such that the position fluctuations at later time can be made small with a proper choice of the parameters, and even smaller than the SQL. The same conclusion that the SQL may not be a lowest limit of the measurement is shown in this mirror-field system.

\section{BACKREACTIONS INDUCED FROM RADIATION PRESSURE}

A stochastic description on dynamics of a mirror moving in quantum fields has been studied in [14]. There the associated semiclassical Langevin equation is derived with the method of Influence Functional by tracing out the quantum fields [14]. This Langevin equation incorporates not only the backreactions but also noises manifested by the field fluctuations. To examine the effect of the backreaction, let us write down the Langevin equation up to first order of the mirror's displacement,

$$
m \ddot{q}(t)-i \int^{t} d t^{\prime} \Theta\left(t-t^{\prime}\right)\left\langle\left[F_{0}(t), F_{0}\left(t^{\prime}\right)\right]\right\rangle_{\bar{\alpha}} q\left(t^{\prime}\right)-\left\langle F_{0}(t)\right\rangle_{\bar{\alpha}}-\left\langle\frac{\delta F}{\delta q}(t)\right\rangle_{\bar{\alpha}} q(t)=\xi_{\bar{\alpha}} .
$$

where $\Theta(\tau)$ is the unit-step function, and the expectation value is taken with respect to the single-mode coherent state $|\bar{\alpha}\rangle$ of the scalar field. There are distinct contributions from the 
quantum scalar field. In addition to the mean radiation pressure given by

$$
\left\langle F_{0}(t)\right\rangle_{\bar{\alpha}}=\frac{1}{2} \int d \mathcal{A}_{\|} \Delta_{\bar{\alpha}}^{z z^{\prime}}\left[z, t ; z^{\prime}, t\right]_{z=z^{\prime}=L}
$$

there are two different backreaction effects [14]. One is the local variation of the mean radiation pressure due to the mean displacement of the mirror,

$$
\left\langle\frac{\delta F}{\delta q}(t)\right\rangle_{\bar{\alpha}} q(t)=\frac{1}{2} \int d \mathcal{A}_{\|} \partial_{L} \Delta_{\bar{\alpha}}^{z z^{\prime}}\left[z, t ; z^{\prime}, t\right]_{z=z^{\prime}=L} q(t)
$$

The other comes from the nonlocal expression, associated with the retarded Green's function, which is constructed from the expectation value of the commutator of the forces. By the Wick's expansion, it can be written in terms of a product of the Wightman functions $\Delta_{\bar{\alpha}}^{z z}$ and $\chi_{0}$ of the scalar field in the case of the single-mode coherent state,

$$
i \Theta\left(t-t^{\prime}\right)\left\langle\left[F(t), F\left(t^{\prime}\right)\right]\right\rangle_{\bar{\alpha}}=\Delta_{\bar{\alpha}}^{z z^{\prime}}\left[z, t ; z^{\prime}, t^{\prime}\right] \chi_{0}\left[z, t ; z^{\prime}, t^{\prime}\right]_{z=L+\epsilon, z^{\prime}=L}
$$

with

$$
\chi_{0}\left[z, t ; z^{\prime}, t^{\prime}\right]=i \Theta\left(t-t^{\prime}\right) \int d \mathcal{A}_{\|} d \mathcal{A}_{\|}^{\prime} \partial_{z} \partial_{z^{\prime}}\left\langle\left[\phi(x), \phi\left(x^{\prime}\right)\right]\right\rangle_{0} .
$$

Here we have used the point-splitting procedure to make this expression well-defined, and will set the small separation parameter $\epsilon$ to zero in the end. In the coordinate representation, these two-point functions take the form

$$
\begin{aligned}
& \Delta_{\bar{\alpha}}^{z z^{\prime}}\left(z, t ; z^{\prime}, t^{\prime}\right)=\left\langle\partial_{z} \phi(x) \partial_{z} \phi\left(x^{\prime}\right)\right\rangle_{\bar{\alpha}} \\
& \quad=\frac{|\bar{\alpha}|^{2} \bar{\omega}}{2 \pi^{3}} \cos [\bar{\omega}(z-L)] \cos \left[\bar{\omega}\left(z^{\prime}-L\right)\right]\left\{\cos \left[\bar{\omega}\left(t-t^{\prime}\right)\right]-\cos \left[\bar{\omega}\left(t+t^{\prime}-2 L\right)-2 \varphi\right]\right\} \\
& \quad i\left\langle\left[\phi(x), \phi\left(x^{\prime}\right)\right]\right\rangle_{0}=\frac{1}{4 \pi R}\left[\delta\left(t-t^{\prime}-R\right)-\delta\left(t-t^{\prime}-\bar{R}\right)\right]-\frac{1}{4 \pi R}\left[\delta\left(t-t^{\prime}+R\right)-\delta\left(t-t^{\prime}+\bar{R}\right)\right],
\end{aligned}
$$

respectively. Here $R$ and $\bar{R}$ are given by

$$
R^{2}=\left(\mathbf{x}_{\|}-\mathbf{x}_{\|}^{\prime}\right)^{2}+\left(z-z^{\prime}\right)^{2}, \quad \bar{R}^{2}=\left(\mathbf{x}_{\|}-\mathbf{x}_{\|}^{\prime}\right)^{2}+\left(z+z^{\prime}-2 L\right)^{2},
$$

and in particular $\bar{R}$ describes the distance between the field point $\left(\mathbf{x}_{\|}, z\right)$ and the image of the source point $\left(\mathbf{x}_{\|}, z^{\prime}\right)$ with respect to the mirror at $z=L$.

After plugging in the appropriate mode functions, the mean radiation pressure and its variation are given by

$$
\begin{aligned}
\langle F(t)\rangle_{\bar{\alpha}} & =\frac{|\bar{\alpha}|^{2}}{4 \pi^{3}} \bar{\omega} \mathcal{A}_{\|}\{1-\cos [2 \bar{\omega}(t-L)-2 \varphi]\}, \\
\left\langle\frac{\delta F}{\delta q}(t)\right\rangle_{\bar{\alpha}} & =-\frac{|\bar{\alpha}|^{2}}{2 \pi^{3}} \bar{\omega}^{2} \mathcal{A}_{\|}\{\sin [2 \bar{\omega}(t-L)-2 \varphi]\} .
\end{aligned}
$$


Although it is oscillatory, the mean radiation pressure of the incoming coherent state is always positive. Thus it keeps pushing the mirror along the positive $z$ axis. Similarly we may carry out the integrals in Eq. 460, and end up with,

$$
\begin{aligned}
& -i \int^{t} d t^{\prime} \Theta\left(t-t^{\prime}\right)\left\langle\left[F(t), F\left(t^{\prime}\right)\right]\right\rangle_{\bar{\alpha}} q\left(t^{\prime}\right) \\
= & -\frac{|\bar{\alpha}|^{2} \bar{\omega}}{4 \pi^{4}} \cos [\bar{\omega}(z-L)] \cos \left[\bar{\omega}\left(z^{\prime}-L\right)\right] \sin [\bar{\omega}(t-L)-\varphi] \int d \mathcal{A}_{\|} d \mathcal{A}_{\|}^{\prime} \\
& \quad \times \int^{t} d t^{\prime} \Theta\left(t-t^{\prime}\right) \sin \left[\bar{\omega}\left(t^{\prime}-L\right)-\varphi\right] q\left(t^{\prime}\right) \partial_{z} \partial_{z^{\prime}}\left[\frac{1}{R} \delta\left(t-t^{\prime}-R\right)-\frac{1}{\bar{R}} \delta\left(t-t^{\prime}-\bar{R}\right)\right] . \\
= & \frac{|\bar{\alpha}|^{2}}{2 \pi^{3}} \bar{\omega} \mathcal{A}_{\|} \sin [\bar{\omega}(t-L)-\varphi]\{\bar{\omega} \cos [\bar{\omega}(t-L)-\varphi] q(t)+\sin [\bar{\omega}(t-L)-\varphi] \dot{q}(t)\},
\end{aligned}
$$

where we have taken the limit $z=z^{\prime}=L$ in the end. The outlines of the calculations can be found in Appendix C. This nonlocal backreaction (50) reduces to local expressions. Especially they include a term proportional to the first-order time derivative of the displacement. Although the phase of the incoming field changes with time, the coefficient of this $\dot{q}(t)$ term remains always positive. Therefore it plays the role of the frictional force, acting against the mirror's motion. It is seen that backreaction effects in Eqs. 499, (50) can be safely ignored as long as $P \bar{\omega} t^{2} / m \ll 1$ in the slow motion approximation, $\bar{\omega} q(t) \ll 1$, as compared with the mean force term in Eq. (48). Some remarks are in order. These backreaction effects not only depend on the mirror's position and velocity but also have time-dependent coefficients. In particular, when the mirror is attached to an extra spring and undergoes oscillatory motion [27], the presence of a time-dependent coefficient of the position term may lead to instabilities on the mirror's dynamics due to parametric oscillation. Then, it might enhance the mirror's response of to small perturbations so as to improve the sensitivity for measurement of the weak signals [22]. Therefore the dynamics of the mirror, when the backreactions to the mirror are included, becomes rich.

The accompanying noise to the backreaction from the commutator of the forces is denoted by $\xi_{\bar{\alpha}}$ in Eq. (43), which is associated with radiation pressure fluctuations,

$$
\xi_{\bar{\alpha}}=F_{0}(t)-\left\langle F_{0}(t)\right\rangle_{\bar{\alpha}},
$$

as has been shown in Eq. (5). The consistent incorporation of these two effects in the Langevin equation relies on the existence of the relation between them, termed as the fluctuations-dissipation relation. For our mirror-field system in the case of single-mode coherent state, this relation can not be presented in a transparent way. Let us write the noise-noise autocorrelation function as

$$
\frac{1}{2}\left\langle\left\{\xi(t), \xi\left(t^{\prime}\right)\right\}\right\rangle_{\bar{\alpha}}=\Delta_{\bar{\alpha}}^{z z^{\prime}}\left[z, t ; z^{\prime}, t^{\prime}\right] \sigma_{0}\left[z, t ; z^{\prime}, t^{\prime}\right]_{z=L+\epsilon, z^{\prime}=L},
$$


where

$$
\sigma_{0}\left[z, t ; z^{\prime}, t^{\prime}\right]=\frac{1}{2} \int d \mathcal{A}_{\|} d \mathcal{A}_{\|}^{\prime} \partial_{z} \partial_{z^{\prime}}\left\langle\left\{\phi(x), \phi\left(x^{\prime}\right)\right\}\right\rangle_{0}
$$

is associated with anti-commutator of the scalar field in its vacuum state. This noisenoise autocorrelation function $(52)$ can then be linked to the retarded Green's function in (46) through a relation between the Fourier transforms of the time-translationally invariant Green's functions $\chi_{0}$ and $\sigma_{0}$. When evaluated at the mirror surface $z=L, z^{\prime}=L$, their Fourier transforms are respectively given by

$$
\begin{aligned}
\chi_{0}\left[z, t ; z^{\prime}, t^{\prime}\right]_{z=L, z^{\prime}=L} & =\int \frac{d w}{2 \pi} \chi_{0}(\omega) e^{-i \omega\left(t-t^{\prime}\right)} \\
\sigma_{0}\left[z, t ; z^{\prime}, t^{\prime}\right]_{z=L, z^{\prime}=L} & =\int \frac{d w}{2 \pi} \sigma_{0}(\omega) e^{-i \omega\left(t-t^{\prime}\right)}
\end{aligned}
$$

and the fluctuations kernel $\sigma_{0}(\omega)$ can be shown to be related to the imaginary part of the $\chi_{0}(\omega)$ kernel,

$$
\sigma_{0}(\omega)=\operatorname{Im}\left[\chi_{0}(\omega)\right][\Theta(\omega)-\Theta(-\omega)] .
$$

Thus we see that the role of the vacuum fluctuations of the field seems to bridge the relation between the noise-noise autocorrelation function and the Green's function constructed by the commutator of the forces so that both effects can be treated in a consistent manner in the Langevin equation.

The backreaction effect has been proposed theoretically in the work of Braginsky [23] for its possible role in setting the sensitivity limit of a detector in the Laser Interferometer Gravitational Wave Observatory (LIGO). Recently, radiation pressure cooling of a micromechanical oscillator has been observed [24]. It raise a hope that quantum noise in a mirror-field system can be further reduced by properly tuning the backreaction effect. In Ref. [12], the backreaction has been extensively studied by modifying the dynamics of the test mass within the "optical spring" scheme to improve performance of the laser interferometer gravitationalwave detector. Here it is shown that these backreactions can be naturally incorporated in the Langevin equation by the field theoretic approach. Hence it will be a necessary next step that we apply the Langevin equation (43) to the interferometer in order to examine quantum noise as well as backreactions.

\section{CONCLUSIONS}

The problem of quantum noise in the mirror-field system has been studied in the field theoretic approach. We consider that a single, perfectly reflecting mirror is illuminated by a single-mode coherent state of a massless scalar field, in addition to ambient vacuum fluctu-

ations. The net field is read out by a monopole detector, placed between the mirror and the 
field source. The radiation pressure of the coherent state drives the mirror into motion. In the slow motion limit, we may identify different sources of quantum noise of the radiation field from readouts of the detector. In turn, the effective distance between the mirror and the detector can be obtained. The sources of quantum noise are respectively attributed to the intrinsic fluctuations of the field (shot noise), induced fluctuations arising from stochastic motion of the mirror due to the radiation pressure fluctuations, and modified field fluctuations which result from the mean displacement of the mirror. Their correlations can then be established resulting from interference between the incident field and the reflected field out of the mirror in the read-out measurement. The overall uncertainty can be found decreased (increased) due to negative (positive) correlation between noise sources. In particular, negative correlation may lead to the situation that overall uncertainty is even lower than that in the standard quantum limit. Backreactions induced by the radiation pressure is studied by deriving the associated Langevin equation from first principles. The backreaction effects are found insignificant for a slowly moving mirror. The Langevin equation incorporates not only backreaction from radiation pressure on the mirror but also noise manifested by the field fluctuations. Equipped with the Langevin equation of the mirror-field system, it deserves further study to improve the performance of the interferometer as the work in [12] by taking advantage of the backreaction effect.

\section{Appendix A: Variation of $I$}

Since we have shown the to the order we are interested, the ordering of operators are irrelevant, we will just write Eq. (13) as

$$
I=I_{0}+q \partial_{L} I_{0}+\frac{1}{2} q^{2} \partial_{L}^{2} I_{0}+\mathcal{O}\left(q^{3}\right)
$$

where the subscript 0 denotes quantities evaluated on the mirror at $z=L$. We further define $\Delta O=O-\langle O\rangle$. Thus A1) up to first order in $\Delta$ becomes

$$
\begin{aligned}
I=\left[\left\langle I_{0}\right\rangle+\Delta I_{0}\right]+ & {\left[(\langle q\rangle+\Delta q)\left(\left\langle\partial_{L} I_{0}\right\rangle+\Delta \partial_{L} I_{0}\right)\right]+\frac{1}{2}\left[(\langle q\rangle+\Delta q)^{2}\left(\left\langle\partial_{L}^{2} I_{0}\right\rangle+\Delta \partial_{L}^{2} I_{0}\right)\right] } \\
=\left[\left\langle I_{0}\right\rangle+\Delta I_{0}\right]+ & {\left[\langle q\rangle\left\langle\partial_{L} I_{0}\right\rangle+\Delta q\left\langle\partial_{L} I_{0}\right\rangle+\Delta \partial_{L} I_{0}\langle q\rangle\right] } \\
& +\frac{1}{2}\left[\langle q\rangle^{2}\left\langle\partial_{L}^{2} I_{0}\right\rangle+2 \Delta q\langle q\rangle\left\langle\partial_{L}^{2} I_{0}\right\rangle+\Delta \partial_{L}^{2} I_{0}\langle q\rangle^{2}\right]+\mathcal{O}\left(\Delta^{2}, q^{3}\right), \quad(\mathrm{A} 2)
\end{aligned}
$$

Taking the expectation value yields

$$
\langle I\rangle=\left\langle I_{0}\right\rangle+\langle q\rangle\left\langle\partial_{L} I_{0}\right\rangle+\frac{1}{2}\langle q\rangle^{2}\left\langle\partial_{L}^{2} I_{0}\right\rangle+\cdots
$$

and thus the quantity $\Delta I$ is given by

$$
\Delta I=\Delta I_{0}+\left[\Delta q\left\langle\partial_{L} I_{0}\right\rangle+\Delta \partial_{L} I_{0}\langle q\rangle\right]+\left[\Delta q\langle q\rangle\left\langle\partial_{L}^{2} I_{0}\right\rangle+\frac{1}{2} \Delta \partial_{L}^{2} I_{0}\langle q\rangle^{2}\right]+\cdots
$$


This is Eq. (16). Since by definition of $\Delta O$, we have $\left\langle(\Delta O)^{2}\right\rangle=\left\langle\Delta^{2} O\right\rangle$; hence the variation of $I$ is

$$
\begin{gathered}
\left\langle\Delta^{2} I\right\rangle=\left\langle\Delta^{2} I_{0}\right\rangle+\left[\left\langle\Delta^{2} q\right\rangle\left\langle\partial_{L} I_{0}\right\rangle^{2}+2\left\langle\Delta q \Delta \partial_{L} I_{0}\right\rangle\langle q\rangle\left\langle\partial_{L} I_{0}\right\rangle+\left\langle\Delta^{2}\left(\partial_{L} I_{0}\right)\right\rangle\langle q\rangle^{2}\right] \\
+\left[2\left\langle\Delta I_{0} \Delta q\right\rangle\left\langle\partial_{L} I_{0}\right\rangle+2\left\langle\Delta I_{0} \Delta \partial_{L} I_{0}\right\rangle\langle q\rangle\right] \\
+\left[2\left\langle\Delta I_{0} \Delta q\right\rangle\langle q\rangle\left\langle\partial_{L}^{2} I_{0}\right\rangle+2\left\langle\Delta I_{0} \Delta \partial_{L}^{2} I_{0}\right\rangle\langle q\rangle^{2}\right]+\cdots,
\end{gathered}
$$

where we have disregarded terms of the order $q^{2} \Delta q$ and higher because the motion of the mirror is minute in comparison with other length scales like $L, z_{0}$ and $\bar{\omega}^{-1}$.

\section{Appendix B: Evaluation of the subdominant terms in $\left\langle\Delta^{2} q\right\rangle_{\bar{\alpha}}$}

The displacement $q(t)$ of the mirror from its original position $z=L$ can be expressed as

$$
q(t)=\frac{1}{m} \int_{0}^{t} d s \int_{0}^{s} d s^{\prime} \int d \mathcal{A}_{\|}: T_{z z}:\left.\left(\mathbf{x}_{\|}, z, s^{\prime}\right)\right|_{z=L},
$$

where the $z z$ component of the normal-ordered stress tensor $: T_{z z}:\left(\mathbf{x}_{\|}, z, t\right)$ is given by

$$
\begin{aligned}
: T_{z z}:\left(x_{\|}, z, t\right)=\frac{1}{2} & \lim _{x^{\prime} \rightarrow x} \partial_{z} \partial_{z}^{\prime} \int^{\prime} \frac{d^{3} k}{(2 \pi)^{\frac{3}{2}}} \frac{1}{\sqrt{2 \omega}} \int^{\prime} \frac{d^{3} k^{\prime}}{(2 \pi)^{\frac{3}{2}}} \frac{1}{\sqrt{2 \omega^{\prime}}} \\
\times & \left\{\mathcal{U}_{\mathbf{k}}\left(\mathbf{x}_{\|}, z, t\right) \mathcal{U}_{\mathbf{k}^{\prime}}\left(\mathbf{x}_{\|}^{\prime}, z^{\prime}, t^{\prime}\right) a_{k} a_{k^{\prime}}+\mathcal{U}_{\mathbf{k}}^{*}\left(\mathbf{x}_{\|}, z, t\right) \mathcal{U}_{\mathbf{k}^{\prime}}^{*}\left(\mathbf{x}_{\|}^{\prime}, z^{\prime}, t^{\prime}\right) a_{k}^{\dagger} a_{k^{\prime}}^{\dagger}\right. \\
& \left.\quad+\mathcal{U}_{\mathbf{k}}^{*}\left(\mathbf{x}_{\|}, z, t\right) \mathcal{U}_{\mathbf{k}^{\prime}}\left(\mathbf{x}_{\|}^{\prime}, z^{\prime}, t^{\prime}\right) a_{k}^{\dagger} a_{k^{\prime}}+\mathcal{U}_{\mathbf{k}}\left(\mathbf{x}_{\|}, z, t\right) \mathcal{U}_{\mathbf{k}^{\prime}}^{*}\left(\mathbf{x}_{\|}^{\prime}, z^{\prime}, t^{\prime}\right) a_{k^{\prime}}^{\dagger} a_{k}\right\},
\end{aligned}
$$

in terms of the mode functions $\mathcal{U}_{\mathbf{k}}\left(\mathbf{x}_{\|}, z, t\right)$. It is convenient to have

$$
\begin{aligned}
& \left.\partial_{z} \mathcal{U}_{\mathbf{k}}\left(\mathbf{x}_{\|}, z, t\right)\right|_{z=L}=\left(2 i k_{z}\right) e^{i \mathbf{k}_{\|} \cdot \mathbf{x}_{\|}-i \omega t} e^{i k_{z} L} \\
& \left.\partial_{z} \mathcal{U}_{\mathbf{k}}^{*}\left(\mathbf{x}_{\|}, z, t\right)\right|_{z=L}=\left(-2 i k_{z}\right) e^{-i \mathbf{k}_{\|} \cdot \mathbf{x}_{\|}+i \omega t} e^{-i k_{z} L}
\end{aligned}
$$

and define

$$
\begin{aligned}
f_{\mathbf{k k}^{\prime}}\left(\mathbf{x}_{\|}, t\right) & =\partial_{z} \mathcal{U}_{\mathbf{k}}^{*}\left(\mathbf{x}_{\|}, z, t\right) \times\left.\partial_{z^{\prime}} \mathcal{U}_{\mathbf{k}^{\prime}}\left(\mathbf{x}_{\|}^{\prime}, z^{\prime}, t^{\prime}\right)\right|_{z=z^{\prime}=L} ^{x^{\prime}=x} \\
& =4 k_{z} k_{z}^{\prime} e^{-i\left(\mathbf{k}_{\|}-\mathbf{k}_{\|}^{\prime}\right) \cdot \mathbf{x}_{\|}+i\left(\omega-\omega^{\prime}\right) t} e^{-i\left(k_{z}-k_{z}^{\prime}\right) L} \\
& =f_{\mathbf{k}^{\prime} \mathbf{k}}^{*}\left(\mathbf{x}_{\|}, t\right), \\
g_{\mathbf{k k}^{\prime}}\left(\mathbf{x}_{\|}, t\right) & =\partial_{z} \mathcal{U}_{\mathbf{k}}\left(\mathbf{x}_{\|}, z, t\right) \times\left.\partial_{z^{\prime}} \mathcal{U}_{\mathbf{k}^{\prime}}\left(\mathbf{x}_{\|}^{\prime}, z^{\prime}, t^{\prime}\right)\right|_{z=z^{\prime}=L} ^{x^{\prime}=x} \\
& =-4 k_{z} k_{z}^{\prime} e^{i\left(\mathbf{k}_{\|}+\mathbf{k}_{\|}^{\prime}\right) \cdot \mathbf{x}_{\|}-i\left(\omega+\omega^{\prime}\right) t} e^{i\left(k_{z}+k_{z}^{\prime}\right) L} \\
& =g_{\mathbf{k}^{\prime} \mathbf{k}}\left(\mathbf{x}_{\|}, t\right) .
\end{aligned}
$$

The displacement operator $q(t)$ then takes a simpler form

$q(t)=\frac{1}{2 m} \int_{0}^{t} d s \int_{0}^{s} d s^{\prime} \int d \mathcal{A}_{\|} \int^{\prime} \frac{d^{3} k}{(2 \pi)^{\frac{3}{2}}} \frac{1}{\sqrt{2 \omega}} \int^{\prime} \frac{d^{3} k^{\prime}}{(2 \pi)^{\frac{3}{2}}} \frac{1}{\sqrt{2 \omega^{\prime}}}\left\{f_{\mathbf{k k}^{\prime}}\left(s^{\prime}\right) a_{\mathbf{k}}^{\dagger} a_{\mathbf{k}^{\prime}}+g_{\mathbf{k k}^{\prime}}\left(s^{\prime}\right) a_{\mathbf{k}} a_{\mathbf{k}^{\prime}}+\right.$ h.c. $\}$.

The calculations of $\left\langle\Delta^{2} q\right\rangle_{\bar{\alpha}}$ involve computations of the expressions like 
- $\left\langle a_{\mathbf{k}}^{\dagger} a_{\mathbf{k}^{\prime}} a_{\tilde{\mathbf{k}}}^{\dagger} a_{\tilde{\mathbf{k}}^{\mathbf{k}^{\prime}}}\right\rangle_{\bar{\alpha}}-\left\langle a_{\mathbf{k}}^{\dagger} a_{\mathbf{k}^{\prime}}\right\rangle_{\bar{\alpha}}\left\langle a_{\tilde{\mathbf{k}}}^{\dagger} a_{\tilde{\mathbf{k}}^{\prime}}\right\rangle_{\bar{\alpha}}$,

- $\left\langle a_{\mathbf{k}} a_{\mathbf{k}^{\prime}} a_{\tilde{\mathbf{k}}}^{\dagger} a_{\tilde{\mathbf{k}}^{\prime}}^{\dagger}\right\rangle_{\bar{\alpha}}-\left\langle a_{\mathbf{k}} a_{\mathbf{k}^{\prime}}\right\rangle_{\bar{\alpha}}\left\langle a_{\tilde{\mathbf{k}}}^{\dagger} a_{\tilde{\mathbf{k}}^{\prime}}^{\dagger}\right\rangle_{\bar{\alpha}}$,

- $\left\langle a_{\mathbf{k}}^{\dagger} a_{\mathbf{k}^{\prime}} a_{\tilde{\mathbf{k}}}^{\dagger} a_{\tilde{\mathbf{k}}^{\prime}}^{\dagger}\right\rangle_{\bar{\alpha}}-\left\langle a_{\mathbf{k}}^{\dagger} a_{\mathbf{k}^{\prime}}\right\rangle_{\bar{\alpha}}\left\langle a_{\tilde{\mathbf{k}}}^{\dagger} a_{\tilde{\mathbf{k}}^{\prime}}^{\dagger}\right\rangle_{\bar{\alpha}},\left\langle a_{\mathbf{k}^{\prime}}^{\dagger} a_{\mathbf{k}} a_{\tilde{\mathbf{k}}}^{\dagger} a_{\tilde{\mathbf{k}}^{\prime}}^{\dagger}\right\rangle_{\bar{\alpha}}-\left\langle a_{\mathbf{k}^{\prime}}^{\dagger} a_{\mathbf{k}}\right\rangle_{\bar{\alpha}}\left\langle a_{\tilde{\mathbf{k}}}^{\dagger} a_{\tilde{\mathbf{k}}^{\prime}}^{\dagger}\right\rangle_{\bar{\alpha}}$, and their complex conjugates,

and so on. The result of the first term is given by Eq. (32). Here we would like to evaluate the second and the third terms, and compare them with the first term.

\section{Evaluation of second term}

Since

$$
\begin{gathered}
\left\langle a_{\mathbf{k}} a_{\mathbf{k}^{\prime}} a_{\tilde{\mathbf{k}}}^{\dagger} a_{\tilde{\mathbf{k}}^{\prime}}^{\dagger}\right\rangle_{\bar{\alpha}}-\left\langle a_{\mathbf{k}} a_{\mathbf{k}^{\prime}}\right\rangle_{\bar{\alpha}}\left\langle a_{\tilde{\mathbf{k}}}^{\dagger} a_{\tilde{\mathbf{k}}^{\prime}}^{\dagger}\right\rangle_{\bar{\alpha}}=|\bar{\alpha}|^{2}\left[\delta(\tilde{\mathbf{k}}-\overline{\mathbf{k}}) \delta\left(\mathbf{k}^{\prime}-\overline{\mathbf{k}}\right) \delta\left(\mathbf{k}-\tilde{\mathbf{k}}^{\prime}\right)+\delta\left(\mathbf{k}^{\prime}-\overline{\mathbf{k}}\right) \delta\left(\tilde{\mathbf{k}}^{\prime}-\overline{\mathbf{k}}\right) \delta(\mathbf{k}-\tilde{\mathbf{k}})\right. \\
\left.+\delta(\mathbf{k}-\overline{\mathbf{k}}) \delta(\tilde{\mathbf{k}}-\overline{\mathbf{k}}) \delta\left(\mathbf{k}^{\prime}-\tilde{\mathbf{k}}^{\prime}\right)+\delta(\mathbf{k}-\overline{\mathbf{k}}) \delta\left(\tilde{\mathbf{k}}^{\prime}-\overline{\mathbf{k}}\right) \delta\left(\mathbf{k}^{\prime}-\tilde{\mathbf{k}}\right)\right] \\
+\delta(\mathbf{k}-\tilde{\mathbf{k}}) \delta\left(\mathbf{k}^{\prime}-\tilde{\mathbf{k}}^{\prime}\right)+\delta\left(\mathbf{k}-\tilde{\mathbf{k}}^{\prime}\right) \delta\left(\mathbf{k}^{\prime}-\tilde{\mathbf{k}}\right)
\end{gathered}
$$

and suppose that the particle number density $|\bar{\alpha}|^{2}$ is sufficiently large such that the pure vacuum contribution can be ignored, the contribution from $\left\langle a_{\mathbf{k}} a_{\mathbf{k}^{\prime}} a_{\tilde{\mathbf{k}}}^{\dagger} a_{\tilde{\mathbf{k}}^{\prime}}^{\dagger}\right\rangle_{\bar{\alpha}}-\left\langle a_{\mathbf{k}} a_{\mathbf{k}^{\prime}}\right\rangle \overline{\bar{\alpha}}\left\langle a_{\tilde{\mathbf{k}}}^{\dagger} a_{\tilde{\mathbf{k}}^{\prime}}^{\dagger}\right\rangle_{\bar{\alpha}}$ to the variance $\left\langle\Delta^{2} q(t)\right\rangle_{\bar{\alpha}}$ is then given by

$$
\begin{aligned}
& \frac{|\bar{\alpha}|^{2}}{m^{2}} \int_{0}^{t} d s \int_{0}^{s} d s^{\prime} \int d \mathcal{A}_{\|} \int_{0}^{t} d \tau \int_{0}^{\tau} d \tau^{\prime} \int d \mathcal{A}_{\|}^{\prime} \int^{\prime} \frac{d^{3} k}{(2 \pi)^{3}} \frac{1}{2 \omega} \frac{1}{(2 \pi)^{3}} \frac{1}{2 \bar{\omega}} g_{\mathbf{k} \overline{\mathbf{k}}}\left(\mathbf{x}_{\|}, s^{\prime}\right) g_{\mathbf{k} \overline{\mathbf{k}}}^{*}\left(\mathbf{x}_{\|}^{\prime}, \tau^{\prime}\right) \\
= & 16 \frac{|\bar{\alpha}|^{2}}{m^{2}} \frac{\mathcal{A}_{\|}}{(2 \pi)^{3}} \frac{\bar{\omega}}{2} \int_{0}^{\infty} \frac{d \omega}{2 \pi} \frac{\omega}{2} \int_{0}^{t} d s \int_{0}^{s} d s^{\prime} \int_{0}^{t} d \tau \int_{0}^{\tau} d \tau^{\prime} e^{-i(\omega+\bar{\omega}) s^{\prime}} e^{+i(\omega+\bar{\omega}) \tau^{\prime}}
\end{aligned}
$$

where we have assumed that the incoming coherent field propagates along the $z$ direction, i.e., $\overline{\mathbf{k}}_{\|}=0$. After integrating the time variables first, we are left with an $\omega$-integral, which is formally UV-divergent. We regularize it with a cutoff frequency $\Lambda$, and then the result of (B2) is

$$
2 \frac{|\bar{\alpha}|^{2} \bar{\omega}}{m^{2} \pi} \frac{\mathcal{A}_{\|} t^{2}}{(2 \pi)^{3}}\left\{\left[\ln \frac{\Lambda+\bar{\omega}}{\bar{\omega}}-\left(1+\frac{\bar{\omega}}{\Lambda}\right)^{-1}\right]+\frac{1}{3 \bar{\omega}^{2} t^{2}}+\mathcal{O}\left(\frac{1}{\bar{\omega}^{3} t^{3}}\right)\right\},
$$

in the long time limit $\bar{\omega} t \gg 1$. The cutoff-dependent term is logarithmic. For any sensible value of $\Lambda$, it should be of the order $\mathcal{O}(1)$. Thus the expressions in the curly brackets are at most of the order unity. Compared with (32), this contribution is of about the order $\mathcal{O}\left(\bar{\omega}^{-1} t^{-1}\right)$ smaller; thus it is relatively subdominant. Note that the contribution from the expression $\left\langle a_{\mathbf{k}}^{\dagger} a_{\mathbf{k}^{\prime}}^{\dagger} a_{\tilde{\mathbf{k}}} a_{\tilde{\mathbf{k}}^{\mathbf{s}^{\prime}}}\right\rangle \overline{\bar{\alpha}}-\left\langle a_{\mathbf{k}}^{\dagger} a_{\mathbf{k}^{\prime}}^{\dagger}\right\rangle_{\bar{\alpha}}\left\langle a_{\tilde{\mathbf{k}}} a_{\tilde{\mathbf{k}}^{\prime}}\right\rangle_{\bar{\alpha}}$ is identically zero for a single-mode coherent field. 


\section{Evaluation of the third therm}

From

$\left\langle a_{\mathbf{k}}^{\dagger} a_{\mathbf{k}^{\prime}} a_{\tilde{\mathbf{k}}}^{\dagger} a_{\tilde{\mathbf{k}}^{\prime}}^{\dagger}\right\rangle_{\bar{\alpha}}-\left\langle a_{\mathbf{k}}^{\dagger} a_{\mathbf{k}^{\prime}}\right\rangle_{\bar{\alpha}}\left\langle a_{\tilde{\mathbf{k}}}^{\dagger} a_{\tilde{\mathbf{k}}^{\prime}}^{\dagger}\right\rangle_{\bar{\alpha}}=\bar{\alpha}^{* 2}\left[\delta(\mathbf{k}-\overline{\mathbf{k}}) \delta(\tilde{\mathbf{k}}-\overline{\mathbf{k}}) \delta\left(\mathbf{k}^{\prime}-\tilde{\mathbf{k}}^{\prime}\right)+\delta(\mathbf{k}-\overline{\mathbf{k}}) \delta\left(\tilde{\mathbf{k}}^{\prime}-\overline{\mathbf{k}}\right) \delta\left(\mathbf{k}^{\prime}-\tilde{\mathbf{k}}\right)\right]$

with $\bar{\alpha}^{*}$ being a complex conjugate of the complex coherent parameter $\bar{\alpha}$, its the contribution to the variance $\left\langle\Delta^{2} q(t)\right\rangle_{\bar{\alpha}}$ is

$$
\begin{aligned}
& \frac{\bar{\alpha}^{* 2}}{2 m^{2}} \int_{0}^{t} d s \int_{0}^{s} d s^{\prime} \int d \mathcal{A}_{\|} \int_{0}^{t} d \tau \int_{0}^{\tau} d \tau^{\prime} \int d \mathcal{A}_{\|}^{\prime} \int^{\prime} \frac{d^{3} k}{(2 \pi)^{3}} \frac{1}{2 \omega} \frac{1}{(2 \pi)^{3}} \frac{1}{2 \bar{\omega}} f_{\overline{\mathbf{k}} \mathbf{k}}\left(\mathbf{x}_{\|}, s^{\prime}\right) g_{\mathbf{k} \mathbf{k}}^{*}\left(\mathbf{x}_{\|}^{\prime}, \tau^{\prime}\right) \\
=- & 8 \frac{\bar{\alpha}^{* 2}}{m^{2}} \frac{\mathcal{A}_{\|}}{(2 \pi)^{3}} \frac{\bar{\omega}}{2} e^{-i 2 \bar{\omega} L} \int_{0}^{\infty} \frac{d \omega}{2 \pi} \frac{\omega}{2} \int_{0}^{t} d s \int_{0}^{s} d s^{\prime} \int_{0}^{t} d \tau \int_{0}^{\tau} d \tau^{\prime} e^{-i(\omega-\bar{\omega}) s^{\prime}} e^{+i(\omega+\bar{\omega}) \tau^{\prime}}
\end{aligned}
$$

We use the following approximations in the long time limit to simplify the $\omega$-integral,

$$
\begin{aligned}
\int_{0}^{t} d s \int_{0}^{s} d s^{\prime} e^{-i(\omega-\bar{\omega}) s^{\prime}} & =2 \frac{\sin ^{2}(\omega-\bar{\omega}) \frac{t}{2}}{(\omega-\bar{\omega})^{2}}-i\left[\frac{t}{\omega-\bar{\omega}}-\frac{\sin (\omega-\bar{\omega}) t}{(\omega-\bar{\omega})^{2}}\right] \\
& \approx 2 \pi \frac{\sin (\omega-\bar{\omega}) \frac{t}{2}}{(\omega-\bar{\omega})} \delta(\omega-\bar{\omega})-i\left[\frac{t}{\omega-\bar{\omega}}-\frac{\sin (\omega-\bar{\omega}) t}{(\omega-\bar{\omega})^{2}}\right] \\
\int_{0}^{t} d \tau \int_{0}^{\tau} d \tau^{\prime} e^{+i(\omega+\bar{\omega}) \tau^{\prime}} & =2 \frac{\sin ^{2}(\omega+\bar{\omega}) \frac{t}{2}}{(\omega+\bar{\omega})^{2}}+i\left[\frac{t}{\omega+\bar{\omega}}-\frac{\sin (\omega+\bar{\omega}) t}{(\omega+\bar{\omega})^{2}}\right] \\
& \approx i\left[\frac{t}{\omega+\bar{\omega}}-\frac{\sin (\omega+\bar{\omega}) t}{(\omega+\bar{\omega})^{2}}\right],
\end{aligned}
$$

due to the fact that $\bar{\omega} t \gg 1$ and $\bar{\omega}>0$. Similar to the previous case, the integral over $\omega$ is divergent, so a cutoff is introduced to regularize the result and Eq. (B3) becomes

$$
-2 \frac{\bar{\alpha}^{* 2} \bar{\omega}}{m^{2} \pi} \frac{\mathcal{A}_{\|} t^{2}}{(2 \pi)^{3}} e^{-i 2 \bar{\omega} L}\left[\ln \frac{\Lambda}{\bar{\omega}}+i \frac{\pi}{4}+\mathcal{O}\left(\frac{1}{\bar{\omega} t}\right)\right] .
$$

There are three other similar terms and their results are either the same as this one or its complex conjugate. Following the arguments in the previous subsection, contributions from this type of expressions are negligible in comparison with 32 in the large time limit. We also note the contribution from $\left\langle a_{\mathbf{k}}^{\dagger} a_{\mathbf{k}^{\prime}}^{\dagger} a_{\tilde{\mathbf{k}}}^{\dagger} a_{\tilde{\mathbf{k}}^{\prime}}\right\rangle_{\bar{\alpha}}-\left\langle a_{\mathbf{k}}^{\dagger} a_{\mathbf{k}^{\prime}}^{\dagger}\right\rangle_{\bar{\alpha}}\left\langle a_{\tilde{\mathbf{k}}}^{\dagger} a_{\tilde{\mathbf{k}}^{\prime}}\right\rangle_{\bar{\alpha}}$ vanishes identically.

As the final remarks, there are no contributions from $\left\langle a_{\mathbf{k}}^{\dagger} a_{\mathbf{k}^{\prime}}^{\dagger} a_{\tilde{\mathbf{k}}}^{\dagger} a_{\tilde{\mathbf{k}}^{\prime}}^{\dagger}\right\rangle_{\bar{\alpha}}-\left\langle a_{\mathbf{k}}^{\dagger} a_{\mathbf{k}^{\prime}}^{\dagger}\right\rangle_{\bar{\alpha}}\left\langle a_{\tilde{\mathbf{k}}}^{\dagger} a_{\tilde{\mathbf{k}}^{\prime}}^{\dagger}\right\rangle_{\bar{\alpha}}$ and $\left\langle a_{\mathbf{k}} a_{\mathbf{k}^{\prime}} a_{\tilde{\mathbf{k}}} a_{\tilde{\mathbf{k}}^{\prime}}\right\rangle_{\bar{\alpha}}-\left\langle a_{\mathbf{k}} a_{\mathbf{k}^{\prime}}\right\rangle_{\bar{\alpha}}\left\langle a_{\tilde{\mathbf{k}}} a_{\tilde{\mathbf{k}}^{\prime}}\right\rangle_{\bar{\alpha}}$ because they vanishes identically, too.

\section{Appendix C: Evaluation of Eq. 50}

We are interested in evaluating part of (50), denoted by $\mathcal{J}$,

$$
\mathcal{J}=\int d \mathcal{A}_{\|} d \mathcal{A}_{\|}^{\prime} \int d t^{\prime} \Theta\left(t-t^{\prime}\right) \sin \left[\bar{\omega}\left(t^{\prime}-L\right)-\varphi\right]\left(\partial_{z} \partial_{z^{\prime}}\left[\phi(x), \phi\left(x^{\prime}\right)\right]\right) \delta q\left(t^{\prime}\right)
$$


The retarded Green's function can be decomposed into two components; one corresponds to unbounded Minkowski space and the other to the image contribution with respect to mirror's original position $z=L$. We consider the unbounded component for the moment, and leave the image contribution to later sections.

Let us take the partial differentiations $\partial_{z}$ before integrals over the cross sectional area $\mathcal{A}_{\|}$. Here we will assume the the linear dimension $\sqrt{\mathcal{A}_{\|}}$is much larger than $\bar{\omega}^{-1}$. Thus the evaluation of $\mathcal{J}$ may proceed as

$$
\begin{aligned}
& \mathcal{J}=\int d \mathcal{A}_{\|} d \mathcal{A}_{\|}^{\prime} \partial_{z} \partial_{z^{\prime}} \int d t^{\prime} \sin \left[\bar{\omega}\left(t^{\prime}-L\right)-\varphi\right] \theta\left(t-t^{\prime}\right) \frac{1}{R} \delta\left(t-t^{\prime}-R\right) q\left(t^{\prime}\right) \\
&=\int d \mathcal{A}_{\|} d \mathcal{A}_{\|}^{\prime} \partial_{z} \partial_{z^{\prime}}\left\{\sin (\vartheta-\bar{\omega} R) \frac{1}{R} q(t-R)\right\}, \quad \vartheta=\bar{\omega}(t-L)-\varphi, \\
&= \sum_{n=0}^{\infty} \frac{(-1)^{n}}{n !} q^{(n)}(t) \int d \mathcal{A}_{\|} d \mathcal{A}_{\|}^{\prime} \partial_{z} \partial_{z^{\prime}}\left\{\sin (\vartheta-\bar{\omega} R) R^{n-1}\right\} \\
&=2 \pi \mathcal{A}_{\|} \sum_{n=0}^{\infty} \frac{(-1)^{n}}{n !} q^{(n)}(t) \int_{|\epsilon|}^{\infty} d R\left\{\bar{\omega}^{2} \epsilon^{2} R^{n-2} \sin (\vartheta-\bar{\omega} R)+\bar{\omega}\left(R^{2}-\epsilon^{2}\right) R^{n-3} \cos (\vartheta-\bar{\omega} R)\right. \\
& \quad+2(n-1) \bar{\omega} \epsilon^{2} R^{n-3} \cos (\vartheta-\bar{\omega} R)-(n-1)\left(R^{2}-\epsilon^{2}\right) R^{n-4} \sin (\vartheta-\bar{\omega} R) \\
&\left.\quad-(n-1)(n-2) \epsilon^{2} R^{n-4} \sin (\vartheta-\bar{\omega} R)\right\},
\end{aligned}
$$

where we have assume that the Taylor's expansion of $q(t-R)$ with respect to $R$ exists, and that implies the assumption that the memory of the system is rather short. We also have used the identities,

$$
\begin{aligned}
\frac{\partial R}{\partial z} & =-\frac{\partial R}{\partial z^{\prime}}=\frac{z-z^{\prime}}{R}=\frac{\epsilon}{R}, \\
\frac{\partial^{2} R}{\partial z^{2}} & =\frac{\partial^{2} R}{\partial z^{\prime 2}}=\frac{1}{R}-\frac{\left(z-z^{\prime}\right)^{2}}{R^{3}}=\frac{1}{R}-\frac{\epsilon^{2}}{R^{3}},
\end{aligned}
$$

with $\epsilon=z-z^{\prime} \lessgtr 0$. For later convenience we will set $\varepsilon=|\epsilon|$, and let $\varepsilon \rightarrow 0$ in the end of the calculations.

First, we check $n=2$ case, and the integral over $R$ becomes

$$
\begin{gathered}
\mathcal{K}_{2}=\int_{\varepsilon}^{\infty} d R\left\{\bar{\omega}^{2} \varepsilon^{2} \sin (\vartheta-\bar{\omega} R)+\bar{\omega}\left(R-\frac{\varepsilon^{2}}{R}\right) \cos (\vartheta-\bar{\omega} R)\right. \\
\left.+2 \bar{\omega} \frac{\varepsilon^{2}}{R} \cos (\vartheta-\bar{\omega} R)-\left(1-\frac{\varepsilon^{2}}{R^{2}}\right) \sin (\vartheta-\bar{\omega} R)\right\} \\
=\int_{\varepsilon}^{\infty} d R\left\{[\bar{\omega} R \cos (\vartheta-\bar{\omega} R)-\sin (\vartheta-\bar{\omega} R)]+\frac{\varepsilon^{2}}{R^{2}}[\bar{\omega} R \cos (\vartheta-\bar{\omega} R)+\sin (\vartheta-\bar{\omega} R)]\right. \\
\left.+\bar{\omega}^{2} \varepsilon^{2} \sin (\vartheta-\bar{\omega} R)\right\}
\end{gathered}
$$


where

$$
\begin{aligned}
\int_{\varepsilon}^{\infty} d R \frac{\varepsilon^{2}}{R^{2}}[\bar{\omega} R \cos (\vartheta-\bar{\omega} R)+\sin (\vartheta-\bar{\omega} R)] & =\varepsilon \sin (\vartheta-\bar{\omega} \varepsilon), \\
\int_{\varepsilon}^{\infty} d R[\bar{\omega} R \cos (\vartheta-\bar{\omega} R)-\sin (\vartheta-\bar{\omega} R)] & =\varepsilon \sin (\vartheta-\bar{\omega} \varepsilon), \\
\int_{\varepsilon}^{\infty} d R \bar{\omega}^{2} \varepsilon^{2} \sin (\vartheta-\bar{\omega} R) & =-\bar{\omega}^{2} \varepsilon \cos (\vartheta-\bar{\omega} \varepsilon) .
\end{aligned}
$$

Thus the integral $\mathcal{K}_{2}$ is of the order $\mathcal{O}(\varepsilon)$ for the $n=2$ case. Note that some of the integrals are still not well-defined in the large $R$ regime so we introduce some cutoff to regularize them, but it turns out that the results are independent of the ultraviolet cutoff.

Similarly for $n=1$, we have

$$
\begin{aligned}
\mathcal{K}_{1} & =\int_{\varepsilon}^{\infty} d R\left\{\bar{\omega}^{2} \frac{\varepsilon^{2}}{R} \sin (\vartheta-\bar{\omega} R)+\bar{\omega}\left(1-\frac{\varepsilon^{2}}{R^{2}}\right) \cos (\vartheta-\bar{\omega} R)\right\} \\
& =\int_{\varepsilon}^{\infty} d R\left\{\bar{\omega} \cos (\vartheta-\bar{\omega} R)+\frac{\bar{\omega} \varepsilon^{2}}{R^{2}}[\bar{\omega} R \sin (\vartheta-\bar{\omega} R)-\cos (\vartheta-\bar{\omega} R)]\right\} \\
& =\int_{\varepsilon}^{\infty} d R \bar{\omega} \cos (\vartheta-\bar{\omega} R)+\mathcal{O}(\varepsilon) \\
& =\sin \vartheta+\mathcal{O}(\varepsilon),
\end{aligned}
$$

where

$$
\int_{\varepsilon}^{\infty} d R \frac{\bar{\omega} \varepsilon^{2}}{R^{2}}[\bar{\omega} R \sin (\vartheta-\bar{\omega} R)-\cos (\vartheta-\bar{\omega} R)]=-\bar{\omega} \varepsilon \cos (\vartheta-\bar{\omega} \varepsilon) .
$$

Finally in the case $n=0$, the integral $\mathcal{K}_{0}$ is given by

$$
\begin{aligned}
\mathcal{K}_{0}=\int_{|\epsilon|}^{\infty} d R\left\{\frac{\bar{\omega}^{2} \epsilon^{2}}{R^{2}} \sin (\vartheta-\bar{\omega} R)+\bar{\omega}\left(\frac{1}{R}-\frac{\epsilon^{2}}{R^{3}}\right) \cos (\vartheta-\bar{\omega} R)-2 \frac{\epsilon^{2}}{R^{4}} \sin (\vartheta-\bar{\omega} R)\right. \\
\left.\quad-2 \frac{\bar{\omega} \epsilon^{2}}{R^{3}} \cos (\vartheta-\bar{\omega} R)+\left(\frac{1}{R^{2}}-\frac{\epsilon^{2}}{R^{4}}\right) \sin (\vartheta-\bar{\omega} R)\right\} \\
=-\bar{\omega} \cos (\vartheta-\bar{\omega} \varepsilon)=-\bar{\omega} \cos \vartheta+\mathcal{O}(\varepsilon) .
\end{aligned}
$$

In general, for $n \geq 2$, it can be shown that

$$
\mathcal{K}_{n}=\varepsilon^{n-1}[-\bar{\omega} \varepsilon \cos (\vartheta-\bar{\omega} \varepsilon)+n \sin (\vartheta-\bar{\omega} \varepsilon)],
$$

so $\mathcal{K}_{n}$ behaves like $\mathcal{O}\left(\varepsilon^{n-1}\right)$ for $n \geq 2$. Therefore $\mathcal{J}$ is

$$
\mathcal{J}=-2 \pi \mathcal{A}_{\|}\{\bar{\omega} \cos \vartheta \delta q(t)+\sin \vartheta \delta \dot{q}(t)+\mathcal{O}(\varepsilon)\} .
$$

\section{Acknowledgments}

DSL would like to thank W. G. Unruh, B. L. Hu and L. H. Ford for stimulating discussions and useful suggestions. This work was supported in part by the National Science Council, 
R. O. C. under grant NSC97-2112-M-259-007-MY3, and the National Center for Theoretical Sciences, Taiwan.

[1] C. M. Caves, K. S. Thorne, R. W. P. Drever, V. D. Sandberg, and M. Zimmermann, Rev. Mod. Phys. 52, 341 (1980).

[2] V. B. Braginsky, Sov. Phys. JETP 26, 831 (1968); V. B. Braginsky and Yu. I. Vorontsov, Sov. Phys. Usp. 17, 644 (1975); V. B. Braginsky, Yu. I. Vorontsov, and F. Ya. Kahili, Sov. Phys. JETP 46, 705 (1977).

[3] C. M. Caves, Phys. Rev. D 23, 1693 (1981).

[4] C. M. Caves, Phys. Rev. Lett. 45, 75 (1980).

[5] H. P. Yuen, Phys. Rev. Lett. 51, 719 (1983); ibid 52, 1730 (1984).

[6] C. M. Caves, Phys. Rev. Lett. 54, 2465 (1985); M. Ozawa, ibid. 60, 385 (1988); Phys. Rev. A 41, 1735 (1990).

[7] R. Loudon, Phys. Rev. Lett. 47, 815 (1981).

[8] W. G. Unruh, in Quantum Optics, Experimental Gravitation, and Measurement Theory, edited by P. Meystre and M. O. Scully (Plenum, New York, 1982), p. 647.

[9] H. J. Kimble, Yuri Levin, Andrey B. Matsko, Kip S. Thorne, and Sergey P. Vyatchanin, Phys. Rev. D 65, 022002 (2001).

[10] M. T. Jaekel and S. Reynaud, Europhys. Lett. 13, 301 (1990); A. Luis and L. L. Sanchez-Soto, Phys. Rev. A 45, 8228 (1992); A. F. Pace, M. J. Collett, and D. F. Walls, Phys. Rev. A 47, 3173 (1993).

[11] V. B. Braginsky, M. L. Gorodetsky, and F. Ya. Khalili, Phys. Lett. A 232, 340 (1997); V. B. Braginsky and F. Ya. Khalili, ibid. 257, 241 (1999).

[12] A. Buonanno and Y. Chen, Phys. Rev. D 64, 042006 (2001); ibid, 65, 042001 (2002).

[13] L. H. Ford and A. Vilenkin, Phys. Rev. D 25, 2569 (1982).

[14] C.-H. Wu and D.-S. Lee, Phys. Rev. D 71, 125005 (2005).

[15] K. Law, Phys. Rev. A, 51, 2537 (1995).

[16] L. H. Ford and T. A. Roman, Sci. Am. 282, No. 1, 30 (2000).

[17] C.-H. Wu and L. H. Ford, Phys. Rev. D, 64, 045010 (2001).

[18] Y. Yamamoto and A. Imamoglu, Mesoscopic Quantum Optics (Wiley, New York, 1990), especially Chap. 3.

[19] J.-T. Hsiang and D.-S. Lee, Phys. Rev. D 73065022 (2006).

[20] J.-T. Hsiang, D.-S. Lee, and C.-H. Wu, J. Korean Phys. Soc. 49, 742 (2006).

[21] J.-T. Hsiang, T.-H. Wu, and D.-S, Lee, Phys. Rev. D 77105021 (2008).

[22] D. Ruger and P. Grutter, Phys. Rev. Lett. 67, 699 (1991).

[23] V. B. Braginsky, S. E. Strigin, and S. P. Vyatchanin, Phys. Lett. A 287, 331 (2001) ; V. B. Braginsky, S. E. Strigin, and S. P. Vyatchanin, Phys. Lett. A 305, 111 (2002).

[24] T. J. Kippenberg, H. Rokhsari, T. Carmon, A. Scherer, and K. J. Vahala, Phys. Rev. Lett. 95, 033901 (2005); A. Schliesser, P. Del-Haye, N. Nooshi, K. J. Vahala, and T. J. Kippenberg, Phys. Rev. Lett 97, 243905 (2006);

[25] T.-H. Wu, J.-T. Hsiang, and D.-S, Lee, in preparation.

[26] M. O. Scully and M. S. Zubairy, Quantum Optics (Cambridge University Press, 2006).

[27] D. Boyanovsky, H. J. de Vega, R. Holman, D.-S. Lee, and A. Singh, Phys. Rev. D 51, 4419 
(1995); D. Boyanovsky, M. D'Attanasio, H. J. de Vega, R. Holman, and D.-S. Lee, Phys. Rev. D 52, 6805 (1995). 